\title{
Selective Inhibition of HER2-Positive Breast Cancer Cells by the HIV Protease Inhibitor Nelfinavir
}

\author{
Joong Sup Shim, Rajini Rao, Kristin Beebe, Len Neckers, Inkyu Han, Rita Nahta, Jun O. Liu \\ Manuscript received July 22, 2011; revised August 8, 2012; accepted August 16, 2012.
}

Correspondence to: Jun O. Liu, PhD, Department of Pharmacology and Molecular Sciences, Johns Hopkins School of Medicine, 725 N Wolfe St, Hunterian Building 516, Baltimore, MD 21205 (e-mail: joliu@jhu.edu).

Background Human epidermal growth factor receptor 2 (HER2)-positive breast cancer is highly aggressive and has higher risk of recurrence than HER2-negative cancer. With few treatment options available, new drug targets specific for HER2-positive breast cancer are needed.

Methods We conducted a pharmacological profiling of seven genotypically distinct breast cancer cell lines using a subset of inhibitors of breast cancer cells from a screen of the Johns Hopkins Drug Library. To identify molecular targets of nelfinavir, identified in the screen as a selective inhibitor of HER2-positive cells, we conducted a genome-wide screen of a haploinsufficiency yeast mutant collection. We evaluated antitumor activity of nelfinavir with xenografts in athymic nude mouse models ( $n=4-6$ per group) of human breast cancer and repeated mixed-effects regression analysis. All statistical tests were two-sided.

Results Pharmacological profiling showed that nelfinavir, an anti-HIV drug, selectively inhibited the growth of HER2positive breast cancer cells in vitro. A genome-wide screening of haploinsufficiency yeast mutants revealed that nelfinavir inhibited heat shock protein 90 (HSP90) function. Further characterization using proteolytic footprinting experiments indicated that nelfinavir inhibited HSP90 in breast cancer cells through a novel mechanism. In vivo, nelfinavir selectively inhibited the growth of HER2-positive breast cancer cells (tumor volume index of HCC1954 cells on day 29 , vehicle vs nelfinavir, mean $=14.42$ vs 5.16 , difference $=9.25,95 \%$ confidence interval $[\mathrm{Cl}]=5.93$ to $12.56, P<.001$; tumor volume index of BT474 cells on day 26 , vehicle vs nelfinavir, mean $=2.21$ vs 0.90 , difference $=1.31,95 \% \mathrm{Cl}=0.83$ to $1.78, P<.001$ ). Moreover, nelfinavir inhibited the growth of trastuzumab- and/or lapatinib-resistant, HER2-positive breast cancer cells in vitro at clinically achievable concentrations.

Conclusion Nelfinavir was found to be a new class of HSP90 inhibitor and can be brought to HER2-breast cancer treatment trials with the same dosage regimen as that used among HIV patients.

J Natl Cancer Inst 2012;104:1576-1590

Breast cancer is one of the leading causes of cancer deaths in the United States. According to the American Cancer Society's most recent estimate, approximately 39520 women died from breast cancer in 2011 (1). Although there are many risk factors known to increase the occurrence of breast cancer, how these risk factors contribute to the transformation of normal cells into cancer cells has remained incompletely understood. Accumulating evidence suggests that genetic alterations, including both inherited and acquired mutations of certain tumor suppressors and oncogenes, are an important cause of breast cancer. For example, inherited mutations in BRCA tumor suppressors confer more than 50\% higher risk for women to develop breast cancer (2). More than 70\% of breast cancer cases with BRCA mutations have the TP53 mutation, and it has been shown that loss of TP53 results in a doubling of breast cancer occurrence in mice with BRCA1 knockout, suggesting that loss of function of these two tumor suppressor genes is a major genetic cause for breast cancer (3).
Estrogen receptor (ER) and human epidermal growth factor receptor 2 (HER2) are both regulated at the level of expression and have served as important diagnostic markers for breast cancer aggressiveness and invasiveness. ER-positive breast cancers tend to grow slowly and have more treatment options (eg, hormonal therapy). In contrast, ER-negative breast cancers can only be treated with chemotherapy (4). HER2 is a member of human epidermal growth factor receptor (EGFR) family consisting of four subtypes, HER1-4 (5). Approximately $25 \%-30 \%$ of human breast cancers overexpress HER2, which is mostly because of amplification of the c-ERBB2 proto-oncogene (6). HER2-positive breast cancer tends to be more aggressive and less responsive to hormone treatments than other types of breast cancer. A few treatments for this type of cancer have been developed, including trastuzumab, a humanized monoclonal antibody (7), and lapatinib, a dual inhibitor of HER2 and EGFR tyrosine kinases that is used in combination with capecitabine (8). 
In addition, a heat shock protein 90 (HSP90) inhibitor, 17-AAG, which is undergoing phase I and II clinical trials for the treatment of lymphomas and solid cancers including metastatic breast cancers (9), has been shown to be effective in HER2-positive breast cancers.

Recently, Vogelstein and colleagues systematically cataloged mutations in a number of breast and colorectal cancer cell lines through genome-wide sequencing of well-annotated human protein-coding genes (10). The knowledge on the genotypic status of each breast cancer cell line offered a unique opportunity to identify genotype-selective anti-breast cancer drugs from our established drug library (Johns Hopkins Drug Library [JHDL]) $(11,12)$. In this study, we screened the JHDL for inhibitors of breast cancer lines and obtained a number of hits, including known anticancer drugs and new ones. Subsequently, we profiled the sensitivity of seven genotypically characterized breast cancer lines to a subset of drugs identified from the JHDL and analyzed intergroup similarity between the drug-sensitivity phenotypes and the defined genotypes of the seven lines, including the mutation status of BRCA and TP53 and the expression status (positive or negative) of ER and HER2. This approach led to the identification of an HIV protease inhibitor, nelfinavir, as an HER2-selective anti-breast cancer drug.

Nelfinavir (Viracept) is an HIV aspartyl protease inhibitor that was approved by the US Food and Drug Administration for the treatment of HIV in 1997 (13). It has been used in combination with other antiretroviral drugs, including HIV reverse-transcriptase inhibitors, for the treatment of HIV infection (14). In addition to its inhibitory effect on HIV, nelfinavir has been shown to inhibit phosphatidylinositol 3-kinase (PI3K) and AKT signaling pathway, a process that is thought to be associated with side effects such as hyperlipidemia (15). Nelfinavir has been reported to inhibit the growth of several types of cancer lines, including melanoma and non-small cell lung cancer $(16,17)$, because of its inhibition of the PI3K and AKT signaling pathway. In addition, the PI3K and AKT pathway is known to be activated after ionizing radiation, conferring resistance to radiotherapy of cancer (18). As a result, inhibition of PI3K and AKT signaling enhances the efficacy of radiation therapy in many types of cancers $(19,20)$. Nelfinavir has entered several clinical trials as either a chemotherapeutic agent or a radiosensitizer for cancer therapy. Promising phase I results have been reported recently for locally advanced pancreatic cancer (21). Despite extensive studies on the anticancer and radiosensitizing activity of nelfinavir, the precise molecular mechanism underlying its anticancer activity and its inhibitory effect on the PI3K and AKT signaling pathway remains unknown. It has been reported that nelfinavir inhibits $20 \mathrm{~S}$ human proteasome activity $(22,23)$, which may account for its inhibition of PI3K and AKT. However, this notion has been questioned (17). In addition, anticancer activity of nelfinavir did not always correlate with the inhibition of PI3K and AKT in vivo (24), suggesting that the PI3K and AKT signaling pathway may not be the only target of nelfinavir in cancer cells.

To further deconvolute the mechanism of action of nelfinavir in HER2-positive breast cancer cells, we screened the entire collection of haploinsufficiency yeast strains and identified HSP90 as a potential target.

\section{Materials and Methods}

\section{Cell Culture and Drug}

HCC1143, HCC1395, HCC1937, HCC1954, HCC2218, MCF7, BT474, and HCC38 breast cancer cells (kindly provided by Dr Bert Vogelstein, Johns Hopkins University School of Medicine, Baltimore, MD) were grown in Roswell Park Memorial Institute 1640 medium containing 10\% fetal bovine serum (Invitrogen, Carlsbad, CA) and 1\% antibiotics (penicillin and streptomycin) solution (Invitrogen). Hs578T cells were grown in Dulbecco's modified Eagle medium with $10 \%$ fetal bovine serum and 1\% antibiotics solution. MCF-10A cells were cultured in Dulbecco's modified Eagle medium: nutrient mixture F12 (Invitrogen) containing $5 \%$ horse serum, $100 \mathrm{ng} / \mathrm{mL}$ cholera toxin, $20 \mathrm{ng} / \mathrm{mL}$ epidermal growth factor (EGF), $0.5 \mu \mathrm{g} / \mathrm{mL}$ hydrocortisone, $10 \mu \mathrm{g} /$ $\mathrm{mL}$ insulin, and $1 \%$ antibiotics solution. Trastuzumab-resistant cells were created by continuously exposing BT474 cells to $4 \mu \mathrm{g} /$ $\mathrm{mL}$ trastuzumab for 3 months, at which point cells regained morphology similar to the parental line (25). Cells per plate were then pooled together and tested for dose response to trastuzumab. Pools are routinely maintained in $4 \mu \mathrm{g} / \mathrm{mL}$ trastuzumab. The cells were maintained in a humidified incubator adjusted to $5 \%$ carbon dioxide. The genotypes of the cell lines were verified using the short tandem repeat profiling by Genetic Resources Core Facility (Johns Hopkins University School of Medicine) as reported previously (12). For drug screening, $10 \mathrm{mM}$ stock solutions of the JHDL were arrayed in 96-well plates and screened at a final concentration of $10 \mu \mathrm{M}$. The cell growth was determined using a $\left[{ }^{3} \mathrm{H}\right]$-thymidine incorporation assay (26). Briefly, cells at 5000 cells per well were seeded in 96-well plates containing $0.2 \mathrm{~mL}$ of growth media and allowed to adhere for 24 hours. The cells were then treated with drugs for 24 hours. Cells were pulsed with $0.5 \mu \mathrm{Ci}\left[{ }^{3} \mathrm{H}\right]$-thymidine (PerkinElmer, Waltham, MA) for 16 hours and harvested upon trypsin treatment onto glass fiber filters (Wallac, Turku, Finland), from which $\left[{ }^{3} \mathrm{H}\right]$ counts were determined using a MicroBeta plate reader (Perkin Elmer).

\section{Drug Sensitivity Profiling}

The half maximal inhibitory concentration $\left(\mathrm{IC}_{50}\right)$ values of 70 drugs against seven breast cancer cell lines were determined using eight different concentrations $(0.01,0.1,0.2,0.5,1,2,5$, and $15 \mu \mathrm{M})$ of each drug by $\left[{ }^{3} \mathrm{H}\right]$-thymidine incorporation assay. The $\mathrm{IC}_{50}$ values were calculated using the GraphPad Prism 4.0 software (GraphPad Software, San Diego, CA). The drug IC $_{50}$ values equal to or less than $10 \mathrm{nM}$ were set as $10 \mathrm{nM}$, and the $\operatorname{drug} \mathrm{IC}_{50}$ values equal to or greater than $15 \mu \mathrm{M}$ were set as $15 \mu \mathrm{M}$. Drug $\mathrm{IC}_{50}$ values $(10-15000 \mathrm{nM})$ were then converted into $\log _{10}$ scale $(1-4.17)$. The four genotypes of seven breast cancer cell lines shown in Supplementary Table 2 (available online) were obtained from the previous report (10) and the National Institutes of Health National Cancer Institute Integrative Cancer Biology Program (http://icbp. lbl.gov/breastcancer/celllines.php) (last accessed August 1, 2012). To examine selective anti-breast cancer drugs by genotype, we used the nonparametric Mann-Whitney $U$ test because measured $\mathrm{IC}_{50}$ values were not normally distributed.

The $\mathrm{IC}_{50}$ values of trastuzumab, lapatinib, and nelfinavir against BT474 and three drug-resistant breast cancer cell lines were determined using eight different concentrations of each drug by 
$\left[{ }^{3} \mathrm{H}\right]$-thymidine incorporation assay. The $\mathrm{IC}_{50}$ values of each drug against each cell line were determined as described above. The resistant index was calculated as a ratio of the drug $\mathrm{IC}_{50}$ against drug-resistant breast cancer cell lines to the $\mathrm{IC}_{50}$ against drug-sensitive cell line BT474.

\section{Screening of Haploinsufficiency Yeasts}

The wild-type (WT) yeast strain BY4743 was used for determining the $\mathrm{IC}_{50}$ value of nelfinavir. For the screening of drug-sensitive strains, the heterozygote yeasts grown in 96-well plates supplemented with the synthetic complete media (MP Biomedicals, Solon, $\mathrm{OH})$ with glucose and $200 \mu \mathrm{g} / \mathrm{mL} \mathrm{G} 418$ were treated with either dimethyl sulfoxide (DMSO) or $10 \mu \mathrm{M}$ nelfinavir $\left(\mathrm{IC}_{10}\right.$ of nelfinavir for WT yeasts) for 24 hours. The yeast growth was measured at an optical density of $600 \mathrm{~nm}$ using a BMG FLUOStar OPTIMA plate reader (BMG Labtech, Cary, NC) to obtain the cell survival rate (\%) of each strain against nelfinavir. The cell survival rate of each strain was then converted into a $Z$ score based on the following equation: $Z$ score $=(x$-average $) /$ standard deviation, where $x$ is the survival rate of each strain against nelfinavir. To collect hits, we used the $Z$ score cutoff of -1.96 (type II error $\alpha=0.05$ ) for the screen. $Z$ scores less than -1.96 gave us $95 \%$ confidence, assuming normal distribution of the yeast viability screening data.

\section{Immunoblot and Immunoprecipitation}

For the immunoblot, cells were lysed by adding 1 volume of $2 \times$ Laemmli buffer and then boiling for 10 minutes. The samples were separated by sodium dodecyl sulfate polyacrylamide gel electrophoresis (SDS-PAGE) and transferred onto nitrocellulose membrane (Bio-Rad, Hercules, CA). Proteins were detected using primary antibodies for HER2 (F-11, Santa Cruz Biotechnology, Santa Cruz, CA; BL3030, Bethyl Labortories, Montgomery, TX), phospho-HER2 (Tyr1248 of human HER2, Santa Cruz Biotechnology), AKT (Santa Cruz Biotechnology), phospho-AKT (Ser473 of mouse AKT, Cell Signaling Technology, Danvers, MA), HSP90 (H-114, Santa Cruz Biotechnology; PA3-013, Thermo Scientific, Rockford, IL), HSP70 (Santa Cruz Biotechnology), extracellular signal-regulated kinase 1 and -2 (ERK1 and -2, Santa Cruz Biotechnology), phospho-ERK1 and -2 (Santa Cruz Biotechnology), caspase-9 (Cell Signaling Technology), caspase-8 (Cell Signaling Technology), caspase-3 (Cell Signaling Technology), HOP (Enzo Life Sciences, Plymouth Meeting, PA), p23 (Abcam, Cambridge, MA), $\alpha$-tubulin (Santa Cruz Biotechnology), cyclindependent kinase 6 (CDK 6) (Santa Cruz Biotechnology), CDK4 (Santa Cruz Biotechnology), activator of heat shock protein ATPase homolog 1 (AHA1) (Santa Cruz Biotechnology), eukaryotic initiation factor $2 \alpha$ (eIF $2 \alpha$ ) (Cell Signaling Technology), phospho-eIF2 $\alpha$ (Enzo Life Sciences), poly ADP ribose polymerase (PARP) (Cell Signaling Technology), and ubiquitin (Santa Cruz Biotechnology); this was followed by incubation with horseradish peroxidase-conjugated antimouse or antirabbit antibodies (Santa Cruz Biotechnology) and enhanced chemiluminescence (GE Healthcare, Pittsburgh, PA). For immunoprecipitation of HSP90 in rabbit reticulocyte lysate (Promega, Madison, WI), $50 \mu \mathrm{L}$ of rabbit reticulocyte lysate were diluted in $450 \mu \mathrm{L}$ of buffer containing $10 \mathrm{mM}$ trisaminomethane hydrochloride, $\mathrm{pH} 7.5,1 \mathrm{mM}$ magnesium chloride, $0.2 \%$ Tween 20 , and $10 \mathrm{mM}$ sodium molybdate and incubated with $4 \mu \mathrm{g}$ HSP90 antibody (H9010, Abcam) overnight at $4^{\circ} \mathrm{C}$. Protein $\mathrm{G}$ sepharose was then added to the sample to precipitate the immunocomplexes for 2 hours. The beads were washed 3 times with the dilution buffer, and the bead samples were processed for SDS-PAGE and immunoblot.

\section{Proteolytic Footprinting of HSP90}

The full-length HSP90a-GST fusion construct (pGEX-4T2-HSP90 $\alpha$ ) was kindly gifted by Dr Solomon Snyder at Johns Hopkins School of Medicine. Each domain of HSP90 $\alpha$ was polymerase chain reaction amplified using primer pairs containing SalI:NotI restriction sites and was subcloned into pGEX-6P-2 vector (GE Healthcare). The GST fusion construct of each domain of HSP90 $\alpha$ was expressed in BL21 and purified by glutathione beads and PreScission protease according to the manufacturer's instruction (GE Healthcare). The full-length HSP90 $\alpha$ (Enzo Life Sciences) or each purified domain was preincubated with drugs for either 30 minutes or 2 hours at room temperature. The reaction mixture was then brought on ice. The trypsin digestion was conducted by incubating the purified HSP90 $\alpha$ and each domain with indicated concentrations of trypsin in an assay buffer containing $10 \mathrm{mM}$ trisaminomethane chloride, $\mathrm{pH} 7.4,150 \mathrm{mM}$ sodium chloride, $4 \mathrm{mM}$ calcium chloride, and $0.1 \mathrm{mM}$ ethylenediaminetetraacetic acid for 10 minutes on ice. The reaction was then stopped by adding 1 volume of $2 \times$ Laemmli buffer, and the samples were boiled for 5 minutes. The proteolytic profile of HSP90 full-length was analyzed by immunoblots with antibodies specific for either the N-terminal (PA3-013) or C-terminal (H-114) of HSP90. The proteolytic profile of HSP90 subdomains were analyzed by SDS-PAGE, followed by Coomassie brilliant blue staining of the gels.

\section{Immunofluorescence}

HCC1954 cells were grown on a glass coverslip in a 24-well plate and were treated with drugs for 24 hours. The cells were fixed with $3.7 \%$ formaldehyde, permeabilized with $0.5 \%$ Triton $\mathrm{X}-100$, and washed with phosphate-buffered saline before being incubated with the blocking solution containing $1 \%$ bovine serum albumin and $0.1 \%$ tween 20 in phosphate-buffered saline for 1 hour. After blocking, the cells were incubated with primary antibodies in the blocking solution overnight at $4^{\circ} \mathrm{C}$, and then incubated with secondary antibodies, including antirabbit immunoglobulin G-cyanine 3 and antimouse immunoglobulin G-cyanine 2, for 1 hour. The cellular nuclei were stained with 4',6-diamidino-2-phenylindole. The immunofluorescence images were obtained using the Zeiss 510 Meta multiphoton confocal microscope (Carl Zeiss, Thornwood, NY).

\section{In Vivo Breast Cancer Xenograft Assays}

Female athymic nude mice (BALB/c, nu/nu-NCr) aged 4-6 weeks and weighing 18-22 $\mathrm{g}$ were purchased from the National Cancer Institute (Frederick, MD) and treated in accordance with Johns Hopkins Animal Care and Use Committee procedures. For the xenograft experiment of HCC1954,HCC1937, and MDA-MB-231, approximately 2 million cells were implanted subcutaneously into mice ( $\mathrm{n}=6$ per group for HCC1954; $\mathrm{n}=4$ per group for HCC1937; n = 5 per group for MDA-MB-231). For the BT474 xenograft, $17 \beta$-estradiol pellets $(0.5 \mathrm{mg}$ per pellet, 60 -day release, 
Innovative Research of America, Sarasota, FL) were implanted at 1 day prior to tumor cell injection. Approximately 6 million BT474 cells were implanted subcutaneously into mice ( $\mathrm{n}=5$ per group). After tumors became palpable, the mice bearing HCC1954 and HCC1937 tumors were treated with either vehicle (saline with 5\% DMSO) or nelfinavir via intraperitoneal injection every day. For BT474 and MDA-MB-231 tumors, mice were given orally either vehicle (saline with $5 \%$ DMSO) or nelfinavir every day. The drug delivery methods and doses of nelfinavir for the mouse experiment were selected based on the previous report (27). Nelfinavir was shown to be effective in growth inhibition of non-small cell lung cancer cell xenograft when mice were treated intraperitoneally with $50 \mathrm{mg} / \mathrm{kg}$ or orally with $100 \mathrm{mg} / \mathrm{kg}$. We used a dose equal to or less than half $(25 \mathrm{mg} / \mathrm{kg}$ for intraperitoneal injection and $40 \mathrm{mg} / \mathrm{kg}$ for oral administration) of the dose used for the non-small cell lung cancer cells. The tumor volume was measured periodically using a vernier caliper and calculated according to the modified ellipsoid formula: tumor volume $\left(\mathrm{mm}^{3}\right)=(\text { short axis })^{2} \times($ long axis $) \times \pi / 6$. After 30 days of treatment, the mice were killed, and the tumor tissues were extracted for immunoblots. The tumor volume index was calculated as a ratio of the tumor volume on a given day divided by the tumor volume of day 0 .

\section{Statistical Analysis}

For in vitro studies, the differences between control and experimental groups were determined by two-sided Student's $t$ test, and $P$ values less than. 05 were considered statistically significant. To evaluate the effects of genotypes of the breast cancer cells on drug sensitivity, we performed the Mann-Whitney $U$ test. For in vivo tumor xenograft studies, a repeated mixed-effects regression analysis was used to evaluate the statistical significance between control and experimental groups over experimental days. The dependent variable was tumor volume index, and the independent variables were the treatment (nelfinavir vs vehicle) and time (experimental days). For each breast cancer xenograft (HCC1954, BT474, HCC1937, and MDA-MB-231), the repeated mixed-effects linear regression model was used to evaluate the effect of nelfinavir on changes in tumor volume indices with an interaction for the categorical variables of treatment (nelfinavir vs vehicle) and experimental days. An autoregressive covariance structure was used to model correlations between the repeated measurements within each treatment with a decay in the strength of correlation depending on the day between measurements. The Akaike information criterion indicated that the autoregressive covariance structure produced an adequate correlation matrix. The overall statistical significance of effects of nelfinavir on changes in tumor volume indices during experimental days was tested using type $3 \mathrm{~F}$ tests. All statistical tests were two-sided.

\section{Results}

\section{Pharmacological Profiling to Identify Genotype-Selective Anti-Breast Cancer Drugs}

To identify genotype-selective anti-breast cancer drugs, we first screened the JHDL for drugs that inhibit breast cancer cell proliferation. Two cell lines, MCF-7, a transformed breast cancer line, and MCF-10A, an immortalized breast epithelial cell line, were used for the initial screening. Drugs that inhibited $\left[{ }^{3} \mathrm{H}\right]$-thymidine incorporation by more than $70 \%$ at $10 \mu \mathrm{M}$ were designated hits. A total of 212 hits were identified from the JHDL, and a subset of 70 drugs was selected to profile seven genotypically characterized breast cancer cell lines based in part on the diversity of drug targets (Supplementary Figure 1, A and B; Supplementary Table 1, available online).

We determined the $\mathrm{IC}_{50}$ value of each of the 70 drugs against the seven genotypically characterized breast cancer cell lines (10) using the $\left[{ }^{3} \mathrm{H}\right]$-thymidine incorporation assay. The $\mathrm{IC}_{50}$ values (ranging $10-15000 \mathrm{nM}$ ) were converted to the $\log _{10}$ scale (ranging $1-4.17$ ) prior to the identification of genotype-selective anti-breast cancer drugs. We were particularly interested in drugs that may have selectivity for mutations in TP53 and BRCA1 and -2, or overexpression of HER2 and ER, given their roles in defining the breast cancer genotype (Supplementary Table 2, available online). We found five drugs, including mercaptopurine, nelfinavir mesylate, gefitinib, triciribine, and $6 \alpha$-methylprednisolone, that showed larger inhibitory potential on cell proliferation with the HER2-positive breast cancer lines than with the HER2-negative breast cancer lines (Table 1). A similar result was obtained from a clustering analysis of standardized drug $\mathrm{IC}_{50}$ values, in which nelfinavir mesylate, gefitinib, and triciribine were clustered in the same group and showed selectivity to HER2positive breast cancer lines (HCC1954 and HCC2218) relative to HER2-negative ones (Supplementary Figure 1, C, available online). Another four drugs were found to be selective for TP53 W'T or mutant cells from the test (Table 1). This result was confirmed again from the clustering analysis (Supplementary Figure 1, C, available online). However, no drugs were found to be selective for BRCA1 and -2 and ER genotypes.

\section{Effect of Nelfinavir on HER2 Signaling Pathway}

Among the five HER2-selective anti-breast cancer drugs identified from the Mann-Whitney $U$ test, $6 \alpha$-methylprednisolone showed the least selectivity for HER2-positive breast cancer cells (Table 1; Supplementary Figure 1, C, available online). Mercaptopurin showed a relatively good selectivity for HER2-positive breast cancer cells, but it also showed a decent inhibitory potential against Hs578T, an HER2-negative cell line (Supplementary Figure 1, C, available online). Therefore, we did not pursue these two drugs further. It is not surprising that gefitinib and triciribine were found to be selective inhibitors for HER2-positive breast cancer cells because gefitinib is an EGFR tyrosine kinase inhibitor (28) and triciribine is an inhibitor of the AKT pathway, which lies downstream of HER2 (29). To determine if other inhibitors of the HER2 signaling pathway also showed selectivity for HER2positive breast cancer cells, we examined inhibitors of PI3K, ERK1 and -2 , and p38 MAPK in several breast cancer cell lines. All the HER2 signaling pathway inhibitors tested here showed a selectivity for HER2-positive breast cancer cells (Supplementary Figure 2, A, available online). Expression levels of HER2 and its downstream pathway proteins in those breast cancer cells were verified by immunoblot analysis (Supplementary Figure 2, B, available online).

Next, we determined the effect of nelfinavir on the HER2 signaling pathway in either HER2-positive (HCC1954 and HCC2218) or HER2-negative (HCC1937; control) breast cancer cells. Nelfinavir $(10 \mu \mathrm{M})$ strongly inhibited all downstream HER2 signaling events examined, including phosphorylation of AKT, ERK1 
Table 1. Nonparametric Mann-Whitney $U$ test of drug sensitivity profiles and breast cancer genotypes*

\begin{tabular}{|c|c|c|c|}
\hline Drugs & $\begin{array}{l}\text { HER2-positive cell lines } \\
\text { Mean } \mathrm{IC}_{50}, \mu \mathrm{M}(95 \% \mathrm{CI})\end{array}$ & $\begin{array}{l}\text { HER2-negative cell lines } \\
\text { Mean } \mathrm{IC}_{50}, \mu \mathrm{M}(95 \% \mathrm{CI})\end{array}$ & Pt \\
\hline Mercaptopurine & $1.75(0.61$ to 2.88$) \dagger$ & $12.75(10.16$ to 15.33$)$ & .002 \\
\hline Nelfinavir mesylate & 3.10 (2.45 to 3.74$)$ & 13.00 (11.91 to 14.08$)$ & .002 \\
\hline Gefitinib & $0.13(0.01$ to 0.28$)$ & 5.46 (4.63 to 6.28$)$ & .002 \\
\hline Triciribine & $0.21(0.01$ to 0.41$)$ & 4.84 (3.01 to 6.67$)$ & .002 \\
\hline $6 \alpha$-methylprednisolone & 0.29 (0.01 to 0.56$)$ & 2.47 (0.57 to 4.38$)$ & .006 \\
\hline Drugs & $\begin{array}{c}\text { TP53 WT cell lines } \\
\text { Mean } \mathrm{IC}_{50}, \mu \mathrm{M}(95 \% \mathrm{CI})\end{array}$ & $\begin{array}{l}\text { TP53 MT cell lines } \\
\text { Mean } \mathrm{IC}_{50}, \mu \mathrm{M}(95 \% \mathrm{CI})\end{array}$ & $P$ \\
\hline Toremifene & 13.00 (9.27 to 16.72$)$ & $15.00(15.00$ to 15.00$) \neq$ & .0003 \\
\hline Phenformin hydrochloride & 2.15 (0.01 to 4.49 ) & 14.66 (14.20 to 15.12$)$ & .003 \\
\hline Proscillaridin A & 0.05 (0.01 to 0.08$)$ & $0.01(0.01$ to 0.02$)$ & .006 \\
\hline Oxaliplatin & 0.05 (0.01 to 0.09$)$ & $0.01(0.01$ to 0.02$)$ & .007 \\
\hline
\end{tabular}

and -2, and the HER2 protein level itself in HER2-positive cells, whereas it had negligible effects on the same events in HER2negative cells (Supplementary Figure 2, C, available online). We noted that the phosphorylation of AKT and ERK1 and -2 eventually succumbed to inhibition by higher concentrations of nelfinavir in HER2-negative breast cancer cells (Figure 1, A). These results suggested that inhibition of AKT and ERK1 and -2 signaling by nelfinavir is, in part, independent of its inhibitory effect on HER2. In addition, nelfinavir activated caspases and induced apoptosis in HCC1954 cells. But no caspase activation or apoptosis occurred in HCC1937 (HER2-negative) or in HCC2218 (HER2-positive), implying that induction of apoptosis by nelfinavir is also independent of its effect on HER2 (Figure 1, A; Supplementary Figure 3, available online). In a time-course experiment with HCC1954 cells, nelfinavir slightly increased the protein level of HER2 in early time points (2-4 hours post-treatment) and then decreased the level of the protein and its phosphorylated form (Figure 1, B). Similar to its effect on HER2, nelfinavir decreased the protein level of AKT as well as its phosphorylated form. Inhibition of HER2 and AKT by nelfinavir was followed by the inhibition of ERK1 and -2 and the induction of apoptosis in HCC1954 cells. These data suggested that nelfinavir might affect the function of a common upstream regulatory protein that is crucial for maintaining the protein levels as well as activities of HER2 and AKT.

The inhibitory effect of nelfinavir on AKT signaling and cancer cell growth has been attributed to its inhibition of the $20 \mathrm{~S}$ proteasome (23). We thus compared the effects of nelfinavir and two well-characterized proteasome inhibitors, MG132 and bortezomib (Velcade), on HER2-positive breast cancer cells. Both nelfinavir and the proteasome inhibitors diminished the HER2 protein level and activated the caspase cascade (Figure 1, C). However, the proteasome inhibitors generated a $23-\mathrm{kD}$ fragment, a putative caspase- 8 cleaved product of HER2, which was not present in cells treated with nelfinavir. In addition, AKT and ERK1 and -2 phosphorylation was greatly inhibited by nelfinavir but not by the proteasome inhibitors. Moreover, caspase inhibitors did not reverse the inhibitory effect of nelfinavir on AKT phosphorylation, whereas the proteasome inhibitors did reverse the nelfinavir effect. In contrast, caspase inhibitors reversed the inhibitory effect of nelfinavir on ERK1 and -2 phosphorylation, whereas the proteasome inhibitors did not. These results demonstrated that inhibition of the proteasome activity cannot account for the biological activity of nelfinavir in breast cancer cells. Given that the proteasome inhibitors prevented certain nelfinavir effects, the actual molecular target of nelfinavir may lie upstream of the proteasome.

\section{Identification of a Molecular Target of Nelfinavir}

We employed the diploid heterozygous deletion mutant yeast collection to screen for synthetic lethal or hypersensitive strains with nelfinavir treatment. Prior to screen, we first analyzed the dose response curve of nelfinavir on the WT diploid yeast (BY4743) (Supplementary Figure 4, A, available online). For the yeast haploinsufficiency screen, we used a suboptimal dose of nelfinavir, which generated only approximately $10 \%$ of maximum inhibition on WT yeast growth $\left(\mathrm{IC}_{10}=10 \mu \mathrm{M}\right)$. This way, we were able to screen a broad range of synthetic lethal strains that showed a hypersensitivity to the low concentration of nelfinavir. From the initial screen, 133 strains were identified to be more sensitive to nelfinavir than WT yeast (Supplementary Figure 4, B and C, available online). We then conducted a secondary screen of the hits and found 17 strains that showed at least two-fold higher sensitivity to nelfinavir than WT yeast showed (Supplementary Table 3, available online). The corresponding gene products include four proteins related to heat shock proteins, five proteins related to Golgi complex and vacuolar proteins, six ribosomal proteins, and two unclassified proteins. Notably, all those proteins are known to interact physically or genetically with HSP82, a yeast ortholog of mammalian HSP90 (Figure 2, A). It is known that deficiency in HSP82 causes synthetic growth defect with those sensitive strains, suggesting that HSP82 might be a potential nelfinavir target in budding yeast and raising the possibility that HSP90 is the target of nelfinavir in mammalian cells.

To assess the possibility that nelfinavir acts on HSP90, we determined the effect of nelfinavir on the interaction between HSP90 and other cochaperones in rabbit reticulocyte lysates using a coimmunoprecipitation assay. We used rabbit reticulocyte 
A

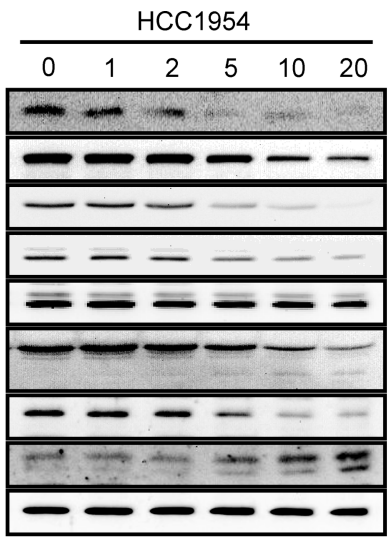

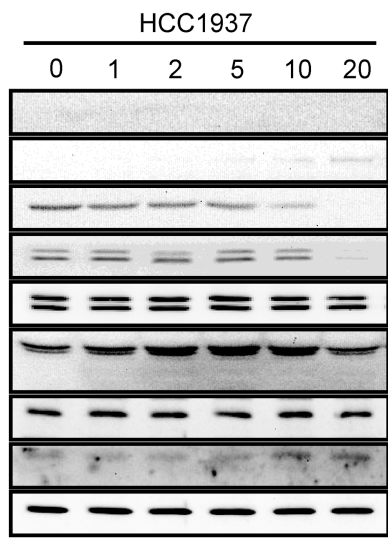

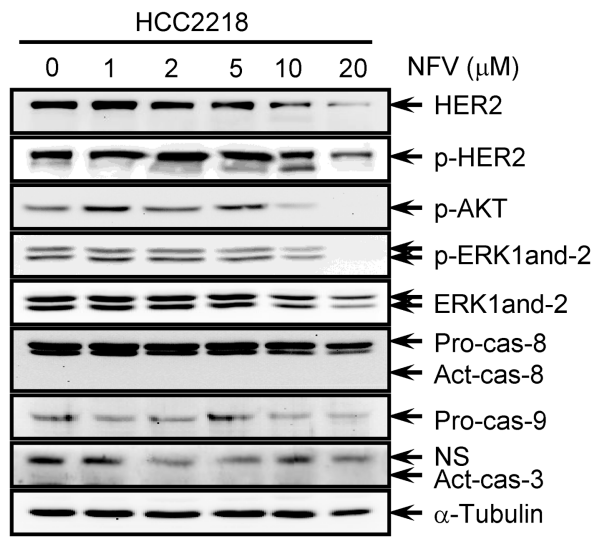

B

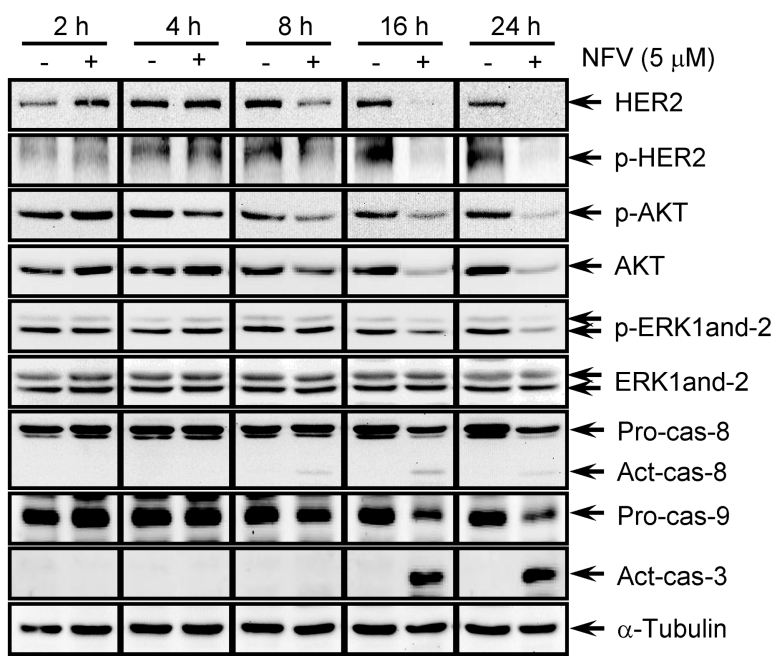

C

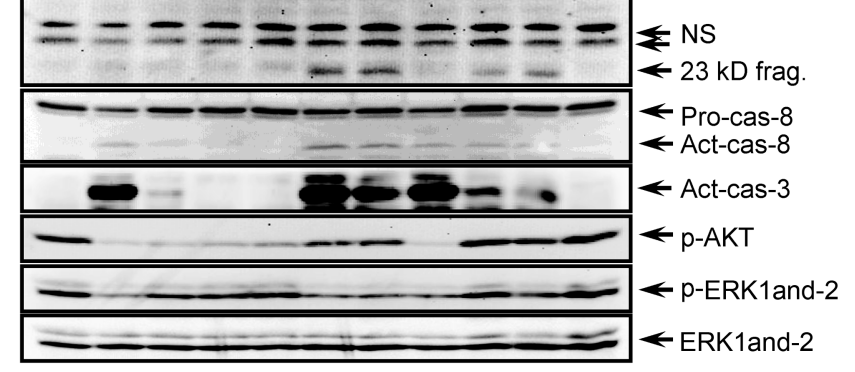

Figure 1. Effect of nelfinavir (NFV) on human epidermal growth factor receptor 2 (HER2) signaling pathway. A) Effect of increasing concentrations of NFV on HER2 signaling pathway and caspase cascades in HER2-positive and -negative breast cancer cells. Cells were treated with various concentrations of NFV for 24 hours, and each protein level was analyzed by immunoblots. B) A time-course experiment of the NFV effect on HER2 signaling pathway and caspase cascades in HER2positive breast cancer, HCC1954 cells. C) Effect of proteasome inhibitors and caspase inhibitors on NFV effects on HER2 signaling pathway and caspase cascades in HCC1954 cells. For the cotreatment experiments,

lysate system for this experiment to avoid any possibility of changes in protein expression levels by drugs. Geldanamycin, an $\mathrm{N}$-terminal binding inhibitor of HSP90, was used as a positive control. Like geldanamycin, nelfinavir enhanced the interaction between HSP70 and HSP90 (Figure 2, B). Unlike geldanamycin, however, nelfinavir had no effect on the interaction between HSP90 and p23. We also examined the effect of nelfinavir on the homodimerization of HSP90, which is known to be inhibited by novobiocin, a C-terminal binding inhibitor of HSP90. Neither nelfinavir nor geldanamycin inhibited HSP90 dimerization and/or oligomerization (Supplementary Figure 5, available online). These results suggested that nelfinavir affects HSP90 function in a way that is distinct from either geldanamycin or novobiocin.

\section{Effect of Nelfinavir on Conformational Changes of HSP90}

HSP90 contains several trypsin cleavage sites (three major and two minor cleavage sites) (Figure 3, A), and trypsin cells were pretreated with either caspase inhibitors or proteasome inhibitors for 30 minutes and then incubated with NFV for 24 hours. z-DEVD (30 $\mu \mathrm{M}$ z-DEVD-fmk, caspase-3 inhibitor), z-IETD (30 $\mu \mathrm{M}$ z-IETDfmk, caspase-8 inhibitor), z-VAD (30 $\mu \mathrm{M}$ z-VAD-fmk, pan caspase inhibitor), MG132 (0.4 $\mu \mathrm{M}$, proteasome inhibitor), and BTZ (20 nM bortezomib, proteasome inhibitor) were used for this study. Act-cas-3 = active form of caspase 3; Act-cas-8 = active form of caspase 8; Act-cas-9 = active form of caspase 9; NS = nonspecific band; pERK1 and -2 ; phospho ERK1 and -2 ; pHER2 = phospho-HER2; Pro-cas-8 = proform of caspase 8 ; Procas-9 $=$ proform of caspase 9 .

digestion profiling (proteolytic footprinting) of HSP90 has been widely used to characterize its drug binding sites as well as its conformational changes (30-32). To determine the effects of nelfinavir and other HSP90 inhibitors on the HSP90 conformational changes, purified HSP90 was incubated with each drug for 2 hours at room temperature before the reaction mixtures were subject to digestion with trypsin for 10 minutes on ice. The reactions were stopped, and the resultant mixtures of HSP90 fragments were analyzed by immunoblot with antibodies directed towards the $\mathrm{N}$ - and C-terminal domains of HSP90, respectively. Both nelfinavir and novobiocin generated increasing amounts of a C-terminal $50 \mathrm{kD}$ (C50) fragment of HSP90 in a dose-dependent manner, whereas DMSO or geldanamycin did not (Figure 3, B). In contrast with novobiocin and geldanamycin, only nelfinavir gave rise to two N-terminal fragments, N78 and N40 of HSP90 (Figure 3, C). These effects of nelfinavir were further confirmed with different concentrations of nelfinavir 
A

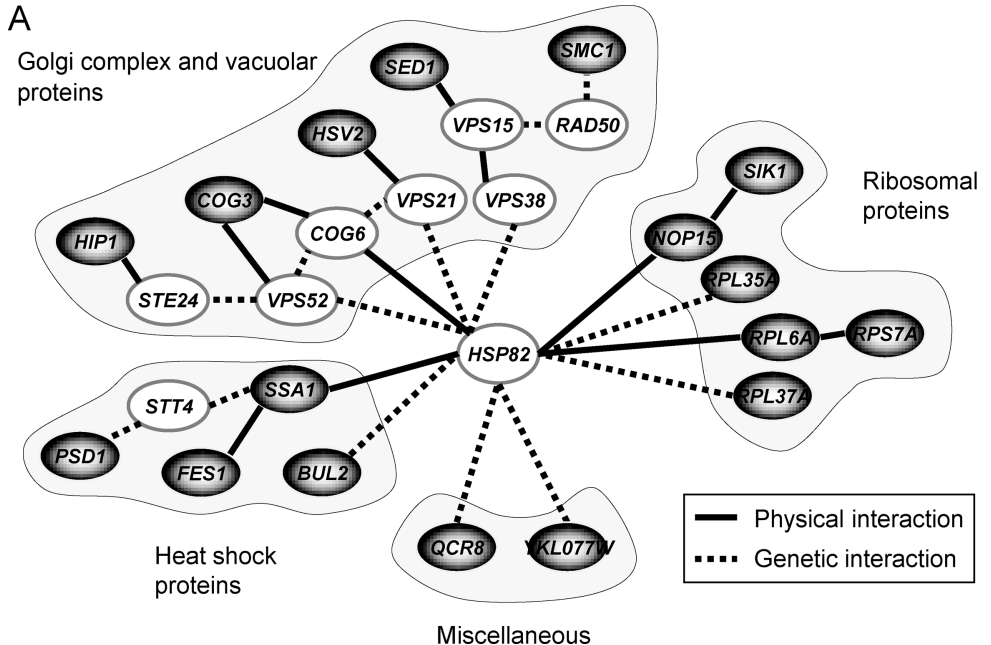

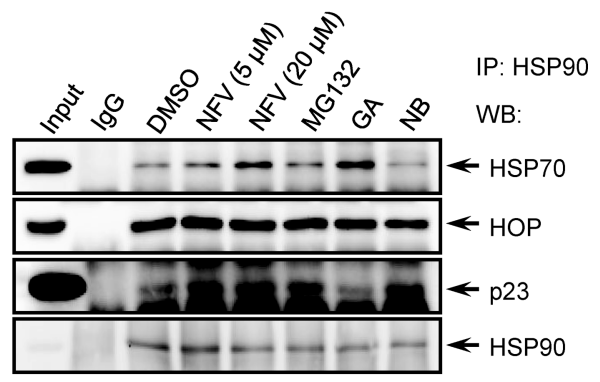

Figure 2. Identification of molecular target of nelfinavir. A) Nelfinavir (NFV) sensitive, heterozygote yeast strains identified from the screen. The yeast strains colored in gray are sensitive to NFV by at least twofold compared with the wild-type parental yeast strain. The yeast strains colored in white, including heat shock protein 82 (HSP82), are genetically or physically linked strains with the NFV-sensitive strains. Solid lines indicate a physical interaction between two genes, and dotted lines represent a genetic interaction. B) Effect of nelfinavir on

(Supplementary Figure 6, available online). We noted that both nelfinavir and novobiocin appeared to have a stabilizing effect on HSP90, presumably due to a small amount of protease contamination during the purification of HSP90, which can slowly digest HSP90 brought to room temperature; this digestion process can be delayed by novobiocin and nelfinavir. We thus incubated a larger amount of HSP90 with drugs for a shorter period of time (30 minutes) and analyzed the trypsin digestion profiles using an HSP90 N-terminal antibody. Interestingly, nelfinavir showed a similar trypsin proteolytic profile to DMSO control under this condition (Figure 3, D). However, the ratio of the amount of $\mathrm{N}$-terminal fragments including N78 and N40 to the full-length HSP90 was increased, suggesting that nelfinavir enhanced the initial trypsin cleavage at 616 and/or 621 and 282 on the full-length HSP90 (Figure 3, D; Figure 4, D). In contrast, novobiocin treatment completely diminished the generation of N78 fragment, demonstrating that it bound around the 616 and/or 621 region and blocked trypsin cleavage on this site. These observations suggested that nelfinavir bound to HSP90 at a site distinct from that of either geldanamycin or novobiocin.

To explore the effect of nelfinavir on HSP90 conformational changes in greater detail, two domains of HSP $90(\mathrm{~N}+\mathrm{M}$ and $\mathrm{M}+\mathrm{C}$, depicted in Figure 3, A) were generated and subjected to the trypsin proteolytic footprinting. Neither novobiocin nor nelfinavir affected the proteolytic profile of $\mathrm{N}+\mathrm{M}$ domain (Figure 3, E). Nelfinavir, however, enhanced the trypsin digestion of the $\mathrm{M}+\mathrm{C}$ domain, with slight increases in the 50 - and the $35-\mathrm{kD}$ fragments, whereas novobiocin delayed the trypsin digestion of $\mathrm{M}+\mathrm{C}$ domain, as judged by an increase in the $50-\mathrm{kD}$ fragment and the corresponding decrease in the $35-\mathrm{kD}$ fragment (Figure 3, F). A time course of the trypsin digestion experiment with the $\mathrm{M}+\mathrm{C}$ domain showed that nelfinavir enhanced the trypsin cleavage of $\mathrm{M}+\mathrm{C}$ domain, with an accumulation of the $50-$ and $35-\mathrm{kD}$ fragments in a time-dependent manner (Figure 4, A and B). The identities interaction between heat shock protein 90 (HSP90) and co-chaperones in rabbit reticulocyte lysates. Rabbit reticulocyte lysates were preincubated with drugs (NFV, $5 \mu \mathrm{M}$ and $20 \mu \mathrm{M}$; MG132, $5 \mu \mathrm{M}$; geldanamycin [GA], $5 \mu \mathrm{M}$; novobiocin [NB], $0.2 \mathrm{mM}$ ) for 2 hours on ice and were diluted in a buffer for the immunoprecipitation assay with HSP9O antibody. DMSO = dimethyl sulfoxide; HOP = HSP70-HSP90 organizing protein; $\mathrm{HSP70}=$ heat shock protein $70 ; \lg \mathrm{G}=$ immunoglobulin $\mathrm{G}$; $\mathrm{IP}=$ immunoprecipitation; $\mathrm{WB}=$ immunoblot.

of both the 50 - and $35-\mathrm{kD}$ fragments were assigned based on the results from the full-length HSP90 and the novobiocin proteolytic profiles of $\mathrm{M}+\mathrm{C}$ domain (Supplementary Figure 7, available online). Because nelfinavir has no effect on the trypsin digestion of the $\mathrm{N}+\mathrm{M}$ domain, it is highly likely that nelfinavir binds to the C-terminal domain of HSP90.

The HSP90 C-terminal fragment contains a dimerization domain that is known to be targeted by novobiocin (33). To determine whether nelfinavir and noboviocin share the same binding site in HSP90, it was sequentially treated with nelfinavir and novobiocin (or vice versa), followed by trypsin digestion. As shown in Figure 4, C, nelfinavir alone significantly enhanced the first cleavage of HSP90 by trypsin, as confirmed by the increase in the ratio of the amount of the tryptic fragments to that of the full-length HSP90. However, regardless of the order of addition, novobiocin completely blocked the effect of nelfinavir on the trypsin digestion profile of HSP90, suggesting that nelfinavir-induced conformational change of HSP90 requires dimerization of HSP90. These results indicate that nelfinavir and novobiocin do not share the same binding site in HSP90 and novobiocin has a dominant effect on the conformational change in the HSP90 over nelfinavir. The overall effects of nelfinavir, geldanamycin, and novobiocin on each trypsin cleavage site are summarized in Figure 4, D. As reported previously, geldanamycin has no effect on the cleavage of HSP90 by trypsin. Nelfinavir seems to bind to the C-terminal region of HSP90 and induce a conformational change that enhanced the initial trypsin cleavage at 282 and 616 and/or 621 sites on the fulllength HSP90 and reduced the cleavage at the 401 site. In contrast, novobiocin binds to $\mathrm{C}$-terminal dimerization domain and induces a conformational change that decreased the trypsin cleavage at 401 and 616 and/or 621 and increased the cleavage at 282 and 227 and/or 250 sites. These observations suggest that although nelfinavir interacts with HSP90 via its C-terminal domain like novobiocin, its binding site differs from that of novobiocin. 


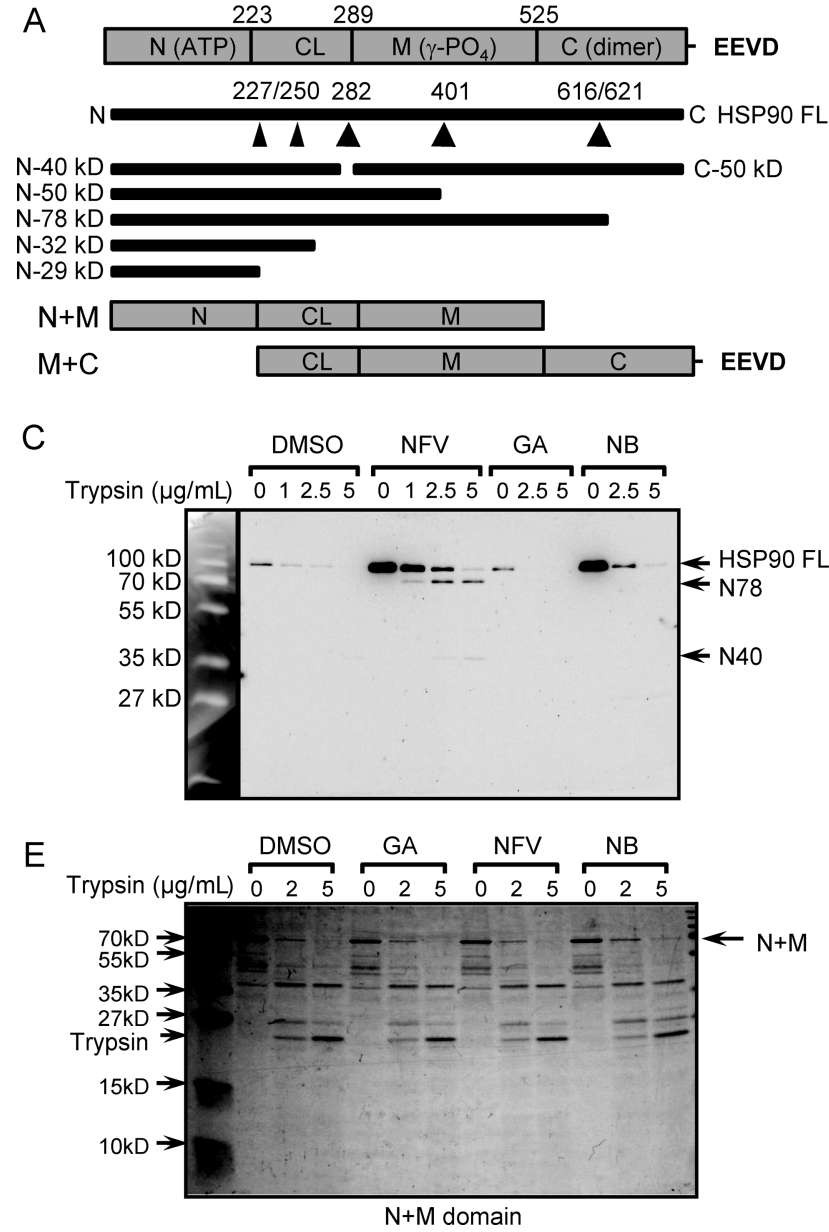

Figure 3. Proteolytic footprinting to assess heat shock protein 90 (HSP90) conformational changes under the nelfinavir (NFV) treatment. A) A schematic illustration of HSP90 domain structures and trypsin cleavage sites. Major peptide fragments of HSP90 generated by trypsin digestion were labeled based on the observed molecular size of each peptide. $\mathrm{N}+\mathrm{M}$ and $\mathrm{M}+\mathrm{C}$ domains of HSP90 were cloned and purified. $\mathbf{B}$ and $\mathbf{C}$ ) Effect of drugs on trypsin digestion profile of the full-length HSP90. Purified full-length (FL) HSP90 (150 ng) was incubated with NFV $(100 \mu \mathrm{M})$, geldanamycin $(\mathrm{GA})(50 \mu \mathrm{M})$, or novobiocin $(\mathrm{NB})(10 \mathrm{mM})$ for 2 hours at room temperature prior to trypsin digestion. The cleavage fragments were then detected using a C-terminal (B) or an N-terminal (C)

\section{Systematic Comparison Between the Effect of Nelfinavir and Known HSP90 Inhibitors on Breast Cancer Cells}

Because nelfinavir has been reported as a human proteasome inhibitor, we verified the effect of nelfinavir on both $20 \mathrm{~S}$ and $26 \mathrm{~S}$ proteasome activity in breast cancer cells. Nelfinavir strongly inhibited the chymotrypsin-like activity of the $20 \mathrm{~S}$ proteasome with an $\mathrm{IC}_{50}$ of $1.5 \mu \mathrm{M}$ (Supplementary Figure 8, A, available online). In contrast, nelfinavir did not affect the $26 \mathrm{~S}$ proteasome activity at all at $20 \mu \mathrm{M}$ concentration in HCC1954 cell lysates. As a control, MG132 completely inhibited the chymotrypsin-like activity and peptidylglutamyl peptide hydrolysing activity of $26 \mathrm{~S}$ proteasome in the lysate (Supplementary Figure 8, B-D, available online). These data demonstrated that nelfinavir has no effect on the $26 \mathrm{~S}$ proteasome activity.

Next, we compared the effects of nelfinavir, some known HSP90 inhibitors, and a proteasome inhibitor on the morphology of HER2-positive breast cancer cells. Both nelfinavir and novobiocin induced a unique morphological change in HCC1954 cells at low
B

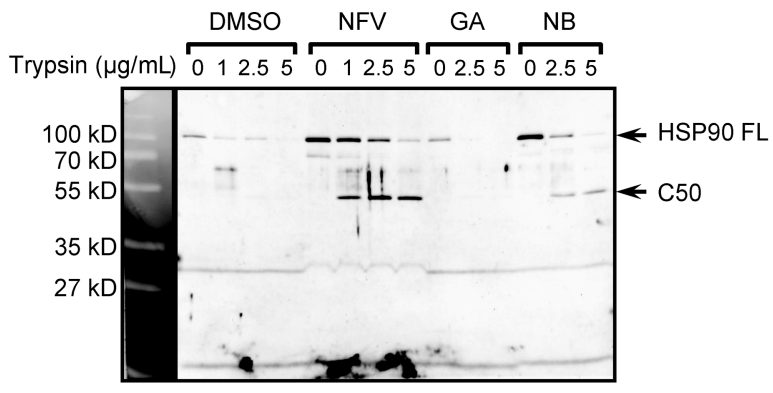

D
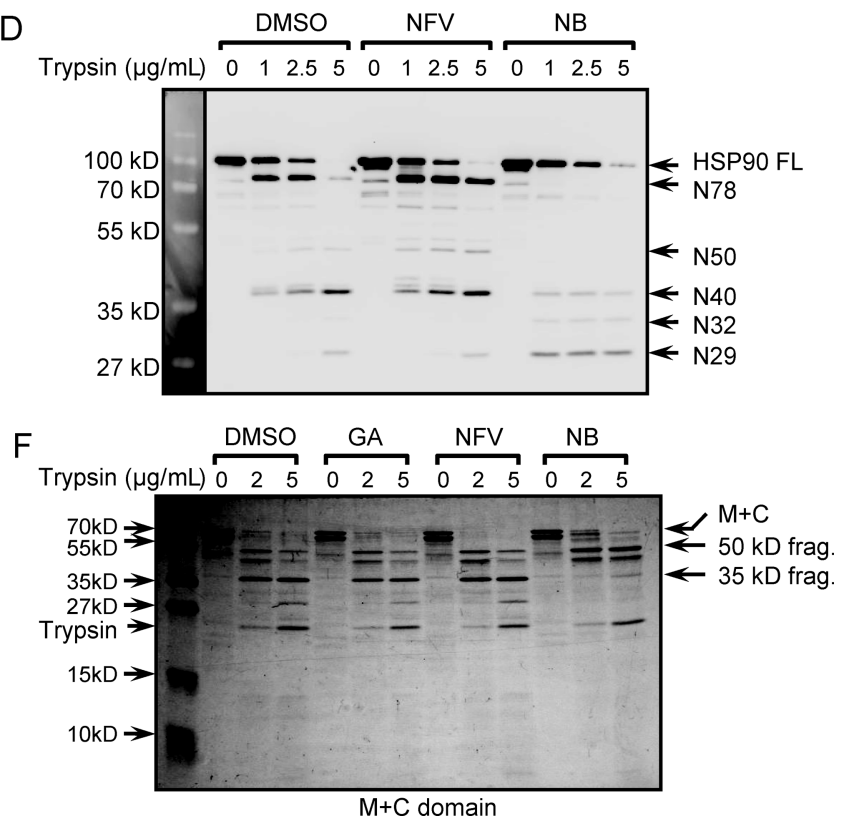

HSP90 antibody. D) FL HSP90 (600 ng) was incubated with NFV (100 $\mu \mathrm{M})$ or NB (10 mM) for 30 minutes at room temperature prior to trypsin digestion. The cleavage fragments were then analyzed using an $\mathrm{N}$-terminal HSP90 antibody. E and F) Effect of drugs on trypsin digestion profile of HSP90 truncation domains. Two micrograms of HSP90 N+M domain (E) or $\mathrm{M}+\mathrm{C}$ domain $(\mathbf{F})$ were incubated with $\mathrm{GA}(50 \mu \mathrm{M})$, NFV $(100 \mu \mathrm{M})$, and $\mathrm{NB}(10 \mathrm{mM})$ for 1 hour at room temperature prior to trypsin digestion. The cleavage products were then analyzed by Coomasie brilliant blue staining after separation with sodium dodecyl sulfate polyacrylamide gel electrophoresis. $\mathrm{CL}=$ charged linker domain; DMSO = dimethyl sulfoxide; EEVD = glutamic acid, glutamic acid, valine, aspartic acid.

drug concentrations and an extensive cellular vacuolization at high concentrations (Supplementary Figure 9, available online). Geldanamycin and MG132 caused quite different morphological changes in the cells. In regard to the HSP90 client proteins, nelfinavir, geldanamycin, and novobiocin decreased the levels of all client proteins examined, whereas MG132 increased the levels of CDKs (Figure 5, A, upper panel). Both nelfinavir and novobiocin reduced the amount of two co-chaperones of HSP90, AHA1 and HSP70, whereas geldanamycin increased the level of those proteins (Figure 5, A, lower panel). As predicted from the induction of the cellular vacuolization, nelfinavir and novobiocin as well as MG132 induced ER stress, as evidenced by the increase in the phosphorylation of eIF2 $\alpha$ (Figure 5, B, upper panel). In contrast, geldanamycin reduced the eIF $2 \alpha$ phosphorylation. Moreover, nelfinavir and novobiocin as well as MG132 strongly enhanced PARP cleavage, a hallmark of apoptosis induction, whereas geldanamycin had a negligible effect. We also analyzed 


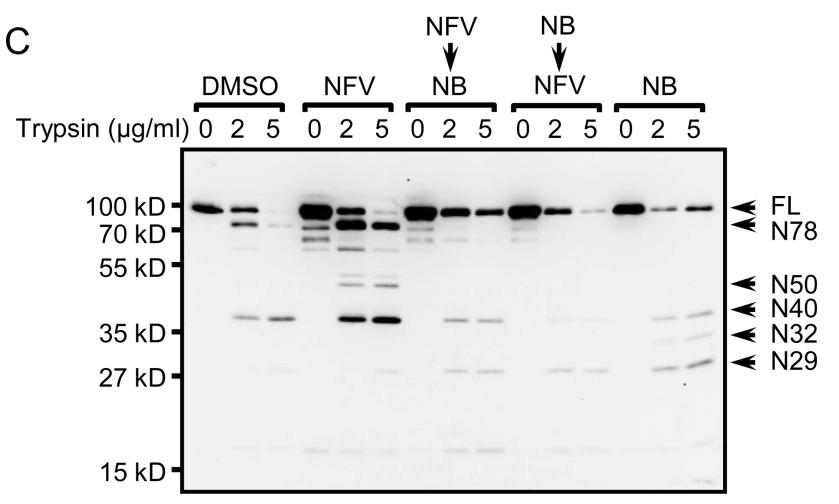

Figure 4. Effect of nelfinavir (NFV) on conformational change of heat shock protein 90 (HSP90) domains. A) A time-course experiment of the trypsin digestion of HSP90 $\mathrm{M}+\mathrm{C}$ domain. The $\mathrm{M}+\mathrm{C}$ domain $(2 \mu \mathrm{g})$ was incubated with dimethyl sulfoxide (DMSO) or NFV for 1 hour at room temperature and digested with trypsin on ice for indicated time points. The cleavage products were then analyzed by Coomasie brilliant blue staining after separation with sodium dodecyl sulfate polyacrylamide gel electrophoresis. Major cleavage fragments, $50 \mathrm{kD}$ and $35 \mathrm{kD}$, are indicated by arrows. A representative gel image from three independent experiments is shown. B) Quantitation of the amount of $\mathrm{M}+\mathrm{C}$ domain and its cleavage products shown in (A). The ratio was calculated by dividing the amount of uncleaved $\mathrm{M}+\mathrm{C}$ domain with the amount of total sum of $\mathrm{M}+\mathrm{C}$ domain, $50 \mathrm{kD}$ fragment and $35 \mathrm{kD}$ fragment. The

the ubiquitination status of cellular proteins after treatment with those inhibitors. Interestingly, both nelfinavir and novobiocin partially induced ubiquitination of proteins, mainly in lower half of all proteins. MG132 strongly increased the ubiquitination of all proteins (Figure 5, B, lower panel). Geldanamycin also slightly increased protein ubiquitination. To confirm the above results visually, we determined the level and subcellular localization of HER2 and ubiquitin in HCC1954 cells by immunofluorescence. As shown in Figure 5, C, HER2 is mainly localized in the cytoplasmic membrane and there is very low basal level of ubiquitinated proteins in the cells. Both nelfinavir and novobiocin reduced the HER2 fluorescence on the cytoplasmic membrane and increased the ubiquitin fluorescence in nuclei with some particles throughout the cytosol, which are presumably preaggresome particles. Geldanamycin strongly reduced the amount of cytoplasmic HER2 and increased protein ubiquitination in nuclei with little cytosolic particles. MG132 reduced the HER2 level and strongly increased ubiquitin fluorescence in nuclei with numerous particles in the cytosol. Finally, we determined the effects of nelfinavir and novobiocin on the interaction between HSP90 and HER2 in HCC1954 cells. In a 3-5-hour treatment during which the HER2

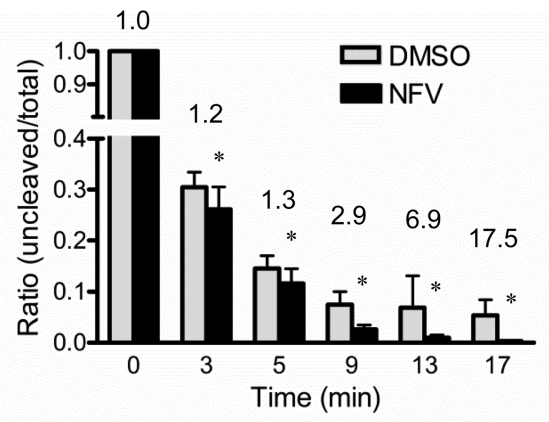

D

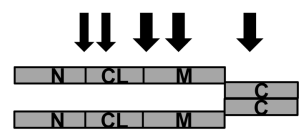

No treatment or GA
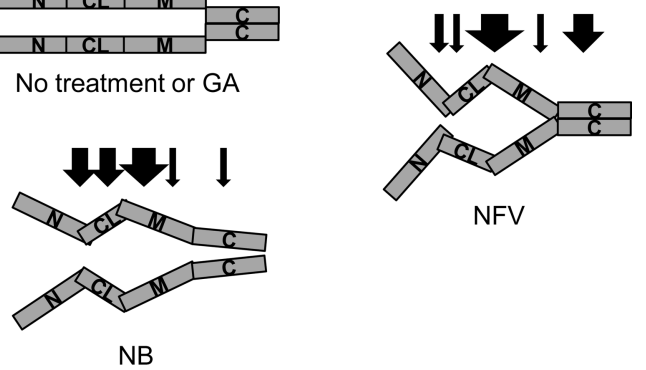

NFV

number on each bar is the relative ratio (average from three independent experiments) between DMSO and NFV. DMSO vs NFV (in each time point), ${ }^{*} P<.05$, calculated by two-sided Student's $t$ test. Error bars $=$ $95 \%$ confidence intervals. C) Effect of cotreatment of NFV and NB (novobiocin) on the trypsin digestion profile of full-length (FL) HSP90. The HSP90 was pretreated with NFV $(100 \mu \mathrm{M})$ or NB $(10 \mathrm{mM})$ for $30 \mathrm{~min}$ utes at room temperature and then treated with NB or NFV for 1 hour at room temperature. The order of treatment is indicated by arrows. The cleavage profile was analyzed by the N-terminal HSP90 antibody. D) Effects of drugs on trypsin digestion profiles of HSP90 are summarized. The trypsin cleavage rate on each site was illustrated with different size of arrows. The bigger arrows are the faster cleavage occurs. $\mathrm{GA}=$ geldanamycin .

protein level was not significantly reduced, both nelfinavir and novobiocin inhibited the interaction between HSP90 and HER2 (Figure 5, D and E).

\section{Effect of Nelfinavir on HER2-Positive and HER2-Negative Breast Cancer Growth in Mice}

We assessed the anti-breast cancer activity of nelfinavir in vivo by paired mouse xenograft experiments with HER2-positive (HCC1954, $\mathrm{n}=6$ per group; BT474, $\mathrm{n}=5$ per group) and HER2negative (HCC1937, $\mathrm{n}=4$ per group; MDA-MB-231, $\mathrm{n}=5$ per group) breast cancer cells. Nude mice bearing tumors were given daily vehicle or nelfinavir for 30 days, and tumor volumes were measured periodically. A longitudinal data analysis showed that intraperitoneal injection of nelfinavir significantly inhibited the xenograft tumor growth of HCC1954 cells over the treatment period (tumor volume index on day 29, vehicle vs nelfinavir, mean $=14.42$ vs 5.16 , difference $=9.25,95 \%$ confidence interval $[\mathrm{CI}]=5.93$ to 12.56 , $P<.001$, two-sided $F$ test), whereas HCC1937 tumor growth was not statistically significantly affected (Figure 6, A and B). Similarly, oral administration of nelfinavir strongly inhibited the tumor growth of BT474 over the treatment period (tumor volume index on day 
A

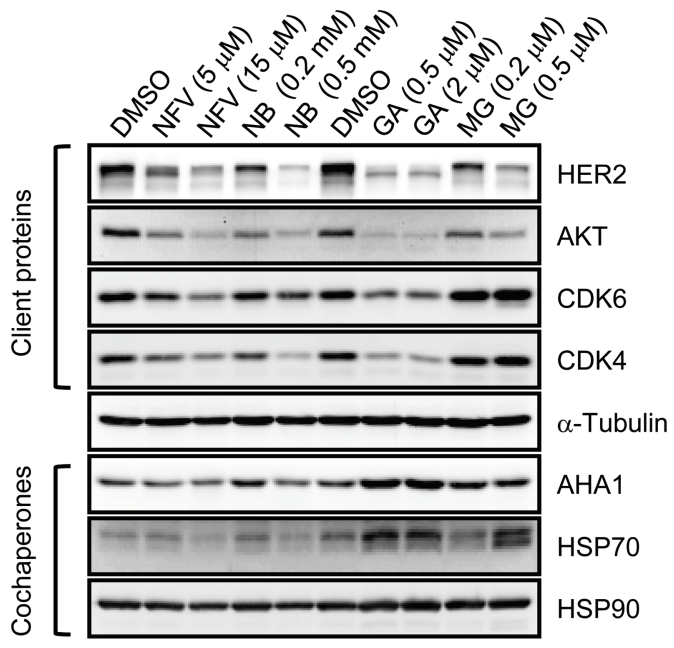

C

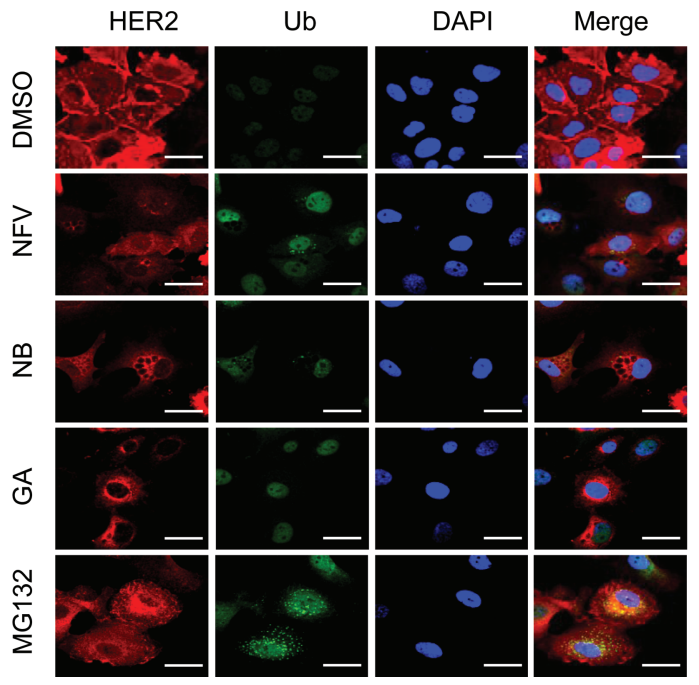

Figure 5. A systematic comparison of nelfinavir (NFV) effect on human epidermal growth factor receptor 2 (HER2)-positive breast cancer cells with that of known heat shock protein 90 (HSP90) inhibitors and a proteasome inhibitor. A and B) Effect of drugs on the protein levels of HSP90 client proteins, co-chaperones, an estrogen receptor (ER) stress marker, an apoptosis marker, and ubiquitinated proteins in HCC1954 cells. The cells were treated with drugs for 24 hours, and immunoblot analyses were conducted. C) Immunofluorescence of HER2 and ubiquitinated proteins in HCC1954 cells, bar $=30 \mu \mathrm{m}$. D and E) Effect of NFV and novobiocin (NB) on interaction between HSP90 and HER2. HCC1954

26 , vehicle vs nelfinavir, mean $=2.21$ vs 0.90 , difference $=1.31,95 \%$ $\mathrm{CI}=0.83$ to $1.78, P<.001$, two-sided $F$ test), whereas the growth of MDA-MB-231 was not statistically significantly affected (Figure 6, $\mathrm{C}$ and $\mathrm{D})$. At the end of the xenograft experiment, the levels of HER2, phospho-HER2, AKT, and phospho-AKT in the tumor tissue extracts were analyzed by immunoblot analysis. Total protein and the phosphorylated levels of HER2 were statistically significantly reduced by nelfinavir in the tumor tissues of BT474 xenografts (Figure 6, E). No detectable HER2 was found in MDA-MB-231 tumor extracts. These results demonstrated that nelfinavir selectively inhibited the growth of HER2-positive breast cancers in vivo and the selective inhibition of HER2-positive breast cancer growth by nelfinavir was attributable to its inhibitory effect on HER2 protein level. Unexpectedly, the levels of AKT and its phosphorylated form were not decreased, but rather, they were increased by nelfinavir in
B

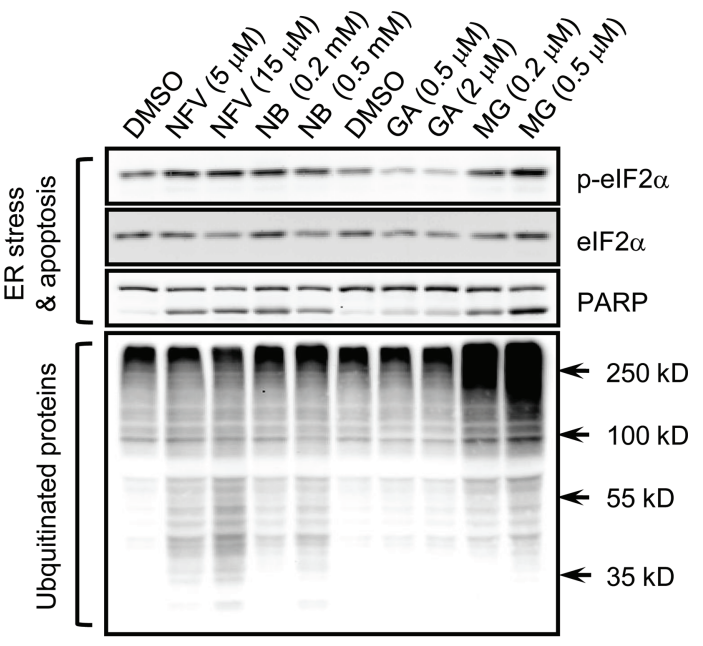

D
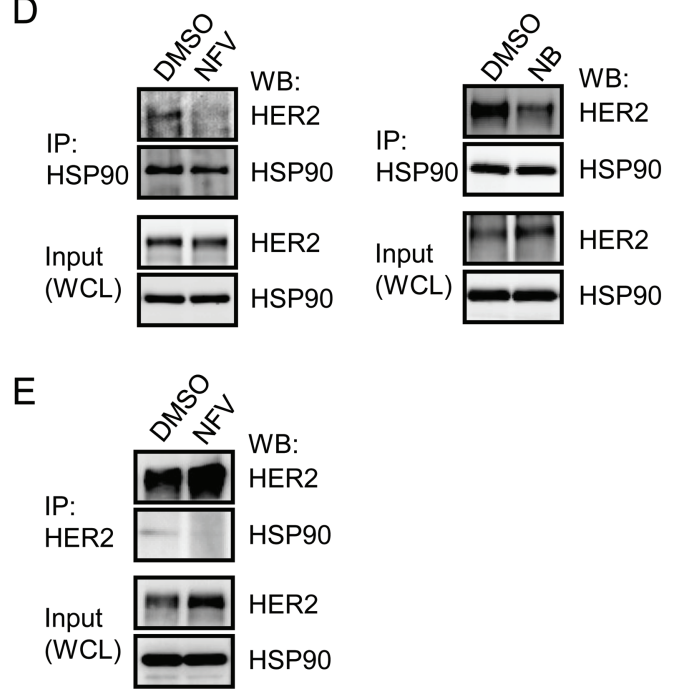

cells were treated with NFV or NB for 5 hours, and HSP90 was immunoprecipitated to analyze the binding status of HER2 (D). The cells were treated with NFV for 3 hours, and HER2 was immunoprecipitated to analyze the binding status of HSP90 (E). Whole cell lysates (WCLs) were used to analyze the input. AHA1 = activator of heat shock protein ATPase homolog 1; CDK4 = cyclin dependent kinase 4 ; CDK6 = cyclin dependent kinase 6; DAPI = 4',6-diamidino-2-phenylindole; elF2 $\alpha=$ eukaryotic initiation factor $2 \alpha$; HSP70 = heat shock protein 70; IP = immunoprecipitation; $\mathrm{GA}=$ geldanamycin; $\mathrm{MG}=\mathrm{MG} 132 ; \mathrm{PARP}=$ poly $\mathrm{ADP}$ ribose polymerase; $\mathrm{Ub}=$ ubiquitin; $\mathrm{WB}=$ immunoblot.

BT474 tumor extracts (Figure 6, E). Also, the levels of AKT and phospho-AKT were not decreased in MDA-MB-231 tumors treated with nelfinavir (Figure 6, F). These results suggest that the inhibitory effect of nelfinavir on PI3K and AKT pathway is not a common mechanism of in vivo anticancer activity of nelfinavir.

\section{Effect of Nelfinavir on the Growth of Drug-Resistant, HER2-Positive Breast Cancer Cells}

We next asked whether nelfinavir as an inhibitor of HSP90 can overcome the drug-resistance of HER2-positive breast cancer cells, which can often occur during treatment with trastuzumab and lapatinib in clinic. We examined both acquired and intrinsic drug-resistant breast cancer cell lines, including BT474-TrastR (acquired trastuzumab resistance), MDA-MB-453 (intrinsic resistance to trastuzumab and lapatinib), and JIMT-1 (intrinsic resistance 

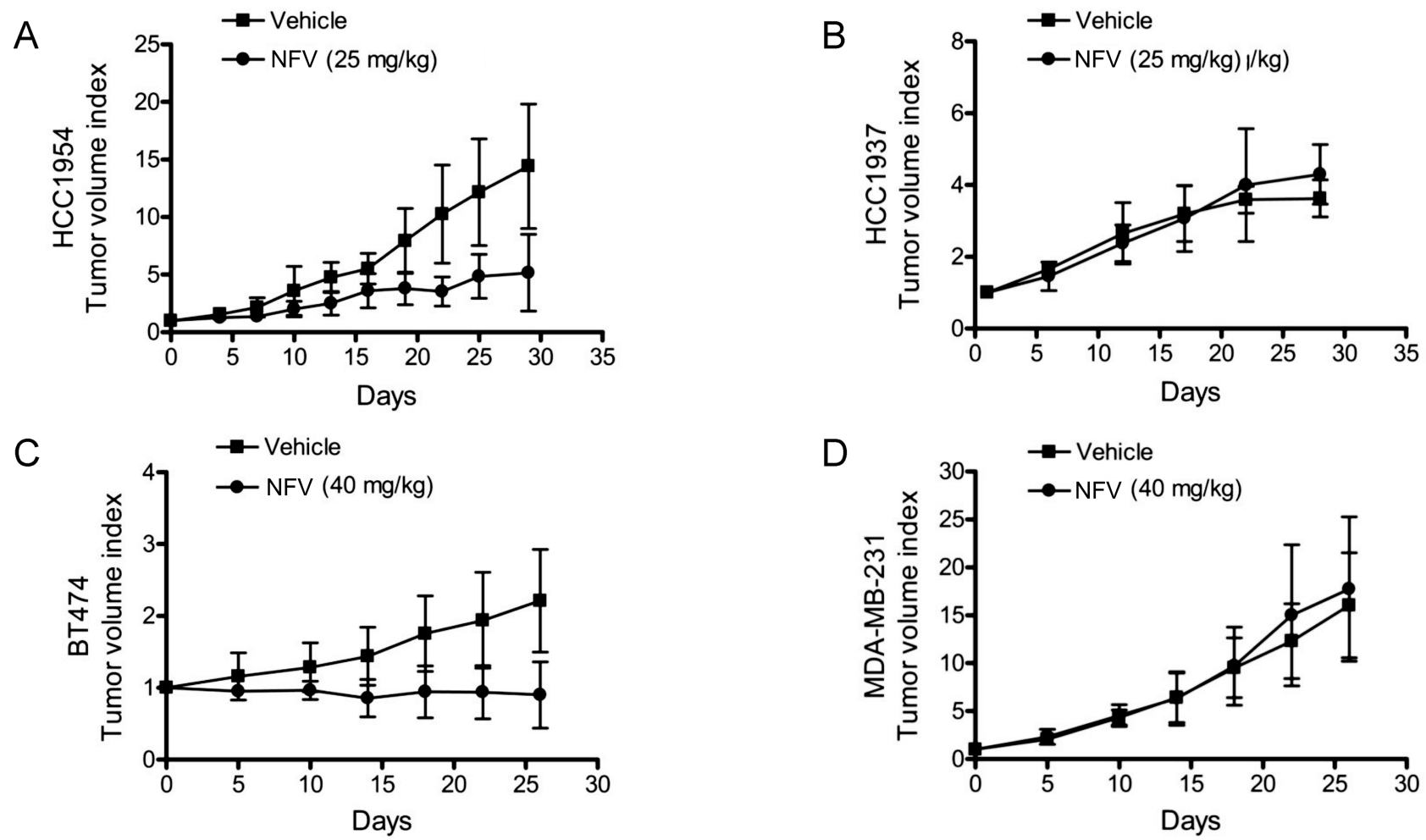

E
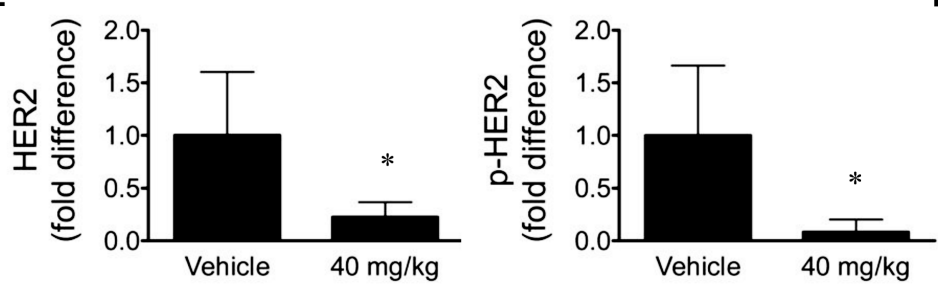

$\mathrm{F}$
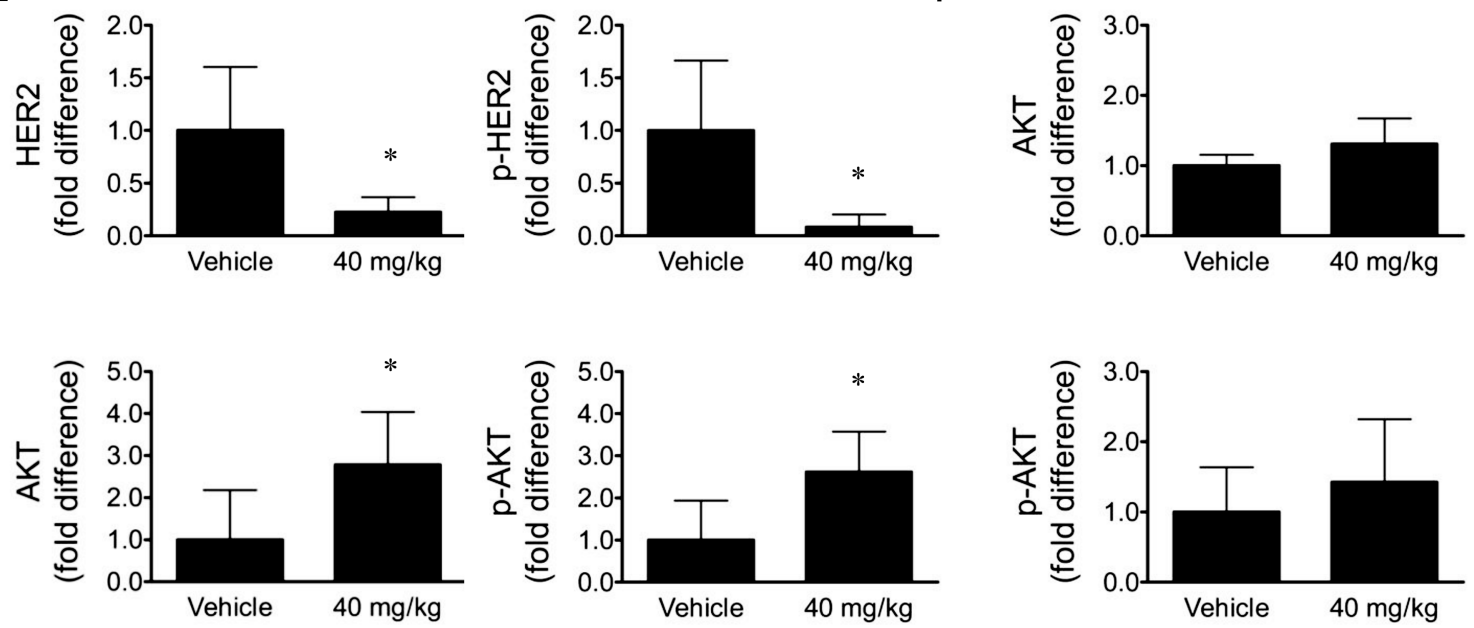

Figure 6. Effect of nelfinavir (NFV) on the growth of human epidermal growth factor receptor 2 (HER2)-positive or HER2-negative breast cancer xenografts in mice. $A$ and B) Analysis of tumor volume index. Twelve female athymic nude mice (BALB/c, nu/nu-NCr) ( $n=6$ mice per group) bearing HCC1954 HER2-positive human breast cancer cells were injected intraperitoneally with vehicle or NFV $(25 \mathrm{mg} / \mathrm{kg})$ every day. Mice bearing HCC1937 HER2-negative breast cancer cells ( $\mathrm{n}=4$ mice per group) were also injected intraperitoneally with vehicle or $\mathrm{NFV}(25 \mathrm{mg} / \mathrm{kg})$ every day. Mean tumor volume indices in each group are shown. The tumor volume index was calculated as a ratio of the tumor volume on a given day divided by the tumor volume of day 0 . Error bars $=95 \%$ confidence intervals. $\mathbf{C}$ and $\mathbf{D}$ ) Analysis of tumor volume

to trastuzumab). BT474 parental cell line was used as a control for drug-sensitive, HER2-positive breast cancer cell line. Trastuzumab inhibited the proliferation of BT474 cells with an $\mathrm{IC}_{50}$ of $1.18 \mu \mathrm{g} /$ $\mathrm{mL}(95 \% \mathrm{CI}=0.89$ to 1.57$)$. However, it didn't inhibit the proliferation of three drug-resistant cell lines up to $50 \mu \mathrm{g} / \mathrm{mL}$ treatment (resistant index > 50) (Figure 7, A; Supplementary Table 4, available online). Lapatinib showed an $\mathrm{IC}_{50}$ value of $0.083 \mu \mathrm{M}$

index. Ten female athymic nude mice (BALB/c, nu/nu-NCr) ( $n=5$ mice per group) bearing BT474 HER2-positive human breast cancer cells or MDA-MB-231 (HER2-negative ones) were orally given vehicle or NFV $(40 \mathrm{mg} / \mathrm{kg})$ every day. Mean tumor volume indices in each group are shown. The tumor volume index was calculated as a ratio of the tumor volume on a given day divided by the tumor volume of day 0 . Error bars $=95 \%$ confidence intervals. $E$ and $\mathbf{F}$ ) The protein levels of HER2, phospho-HER2 (p-HER2), AKT, and phospho-AKT (p-AKT) in the BT474 (E) or MDA-MB-231 (F) tumor extracts were analyzed by immunoblots and quantitated using Image $\mathrm{J}$ software. Vehicle vs test groups, ${ }^{*} P<.05$, calculated by two-sided Student's $t$ test. Error bars $=95 \%$ confidence intervals.

(95\% CI $=0.073$ to 0.093 ) for BT474 cells, but it showed $\mathrm{IC}_{50}$ values of $1.35 \mu \mathrm{M}(95 \% \mathrm{CI}=1.08$ to 1.67 , resistant index $=16.26)$ and $1.07 \mu \mathrm{M}(95 \% \mathrm{CI}=0.74$ to 1.55 , resistant index $=12.89)$ for MDA-MB-453 and JIMT-1 cells, respectively (Figure 7, B; Supplementary Table 4, available online). Nelfinavir inhibited the proliferation of BT474 cells with an $\mathrm{IC}_{50}$ of $5.83 \mu \mathrm{M}(95 \% \mathrm{CI}=5.46$ to 6.22). It also inhibited the proliferation of all three drug-resistant 
A

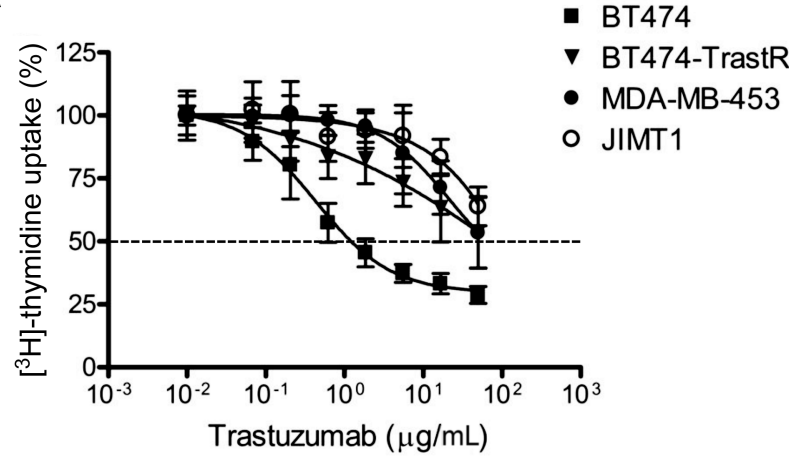

C

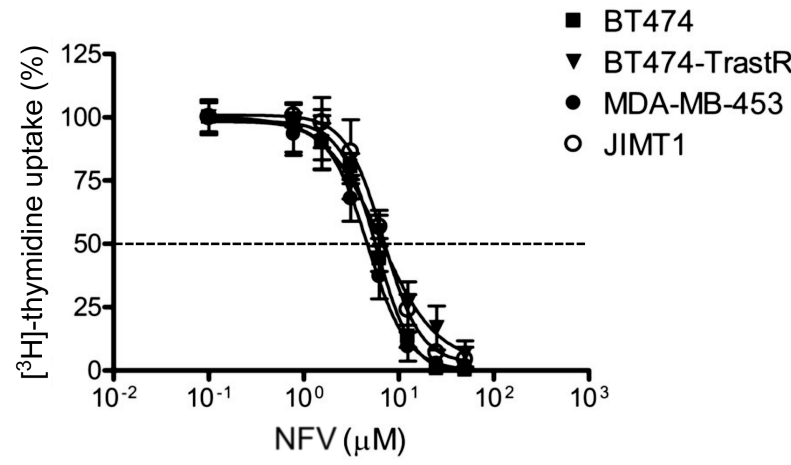

B

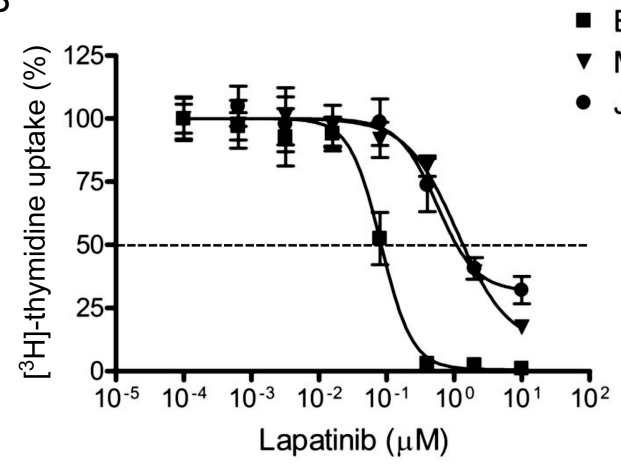

BT474

MDA-MB-453

- JIMT1
Figure 7. Effect of nelfinavir on the proliferation of trastuzumab-/lapatinib-resistant, human epidermal growth factor receptor 2 (HER2)-positive breast cancer cells. Cell proliferation was assessed using $\left[{ }^{3} \mathrm{H}\right]$-thymidine incorporation assay. A) Effect of trastuzumab on the proliferation of drug-sensitive and -resistant breast cancer cells. The dose-response curves of trastuzumab on BT474 (drug-sensitive, Her2-positive breast cancer cell line), BT474-TrastR (acquired trastuzumab-resistant BT474 cells), MDA-MB-453 (intrinsic trastuzumab-resistant, Her2-positive breast cancer cell line), and JIMT1 (intrinsic trastuzumab-resistant,

cell lines within a concentration range that was effective in BT474 ( $\mathrm{IC}_{50}$ values for BT474-TrastR cells: $6.44 \mu \mathrm{M}, 95 \% \mathrm{CI}=5.14$ to 8.07 , resistant index $=1.1$; for MDA-MB-453 cells: $4.74 \mu \mathrm{M}$, $95 \% \mathrm{CI}=4.17$ to 5.38 , resistant index $=0.81$; for JIMT-1 cells: $6.88 \mu \mathrm{M}, 95 \% \mathrm{CI}=6.18$ to 7.66 , resistant index $=1.18$ ) (Figure 7, C; Supplementary Table 4, available online).

\section{Discussion}

Identification and characterization of new pharmacological activities from existing drugs represents an effective way to accelerate the translation of discoveries at the bench to clinical applications; it also facilitates the subsequent target identification and validation. Under these premises, we established the JHDL. To date, a number of interesting and potentially applicable hits have been identified, including the immunosuppressive drug mycophenolic acid, the antifungal drug itraconazole, and the urinary tract antibiotic nitroxoline as novel antiangiogenic agents; the antileprosy drug clofazimine as a novel inhibitor of Kv1.3 channel and immunomodulator; and the antihistamine astemazole as novel antimalarial agent $(11,12,26,34-36)$. In the present study, we performed a two-stage screen for new anti-breast cancer agents. In stage 1 , we screened the entire JHDL against two representative breast cell lines for inhibitors and identified over 200 hits. In stage 2, a subset of the
Her2-positive breast cancer cell line) are shown. The dashed line represents a half maximal inhibitory concentration $\left(\mathrm{IC}_{50}\right)$ of the drug. Error bars $=95 \%$ confidence intervals. B) Effect of lapatinib on the proliferation of drug-sensitive and -resistant breast cancer cells. The dashed line represents an $\mathrm{IC}_{50}$ of the drug. Error bars $=95 \%$ confidence intervals. C) Effect of nelfinavir on the proliferation of drug-sensitive and -resistant breast cancer cells. The dashed line represents an $\mathrm{IC}_{50}$ of the drug. Error bars $=95 \%$ confidence intervals.

hits was profiled against seven genotypically characterized breast cancer cell lines. We identified nelfinavir as a selective inhibitor of HER2-positive breast cancer cells. Selectivity of nelfinavir for HER2-positive breast cancer cells was attributable to its inhibitory effects on HER2 protein and phosphorylation levels as well as AKT and ERK1 and -2 signaling pathways. In vivo anti-breast cancer activities of nelfinavir were verified using mouse xenograft models with both HER2-positive and -negative breast cancer cells. Either intraperitoneal or oral administration of nelfinavir selectively suppressed the growth of HER2-positive breast cancer cells over the HER2-negative ones. Immunoblot analysis confirmed that nelfinavir had a similar effect on HER2 protein level in vivo.

To unravel the mechanistic basis of the selectivity of nelfinavir for HER2-positive breast cancer cells, we initially entertained the possibility that nelfinavir worked through inhibition of the human proteasome, as previously reported (23). Although we were able to confirm the inhibitory effect of nelfinavir on purified $20 \mathrm{~S}$ proteasome, several lines of evidence argued against the proteasome as the relevant target for nelfinavir for its selective inhibition of HER2-positive breast cancer cells. First, pretreatment with proteasome inhibitors blocked certain nelfinavir effects. Second, nelfinavir did not inhibit 26S proteasome activity at all in cell lysates. Third, whereas MG132 increased the protein level of CDK4 and CDK6, nelfinavir enhanced degradation of those CDKs, whose stability is known to be regulated 
by the proteasome-dependent pathway. Lastly, morphological changes of the breast cancer cells caused by nelfinavir were distinct from those induced by MG132. These results suggested that nelfinavir has a different target than the $20 \mathrm{~S}$ proteasome.

We employed a chemical genetic screening of haploinsufficiency yeast collection to seek clues on the potential nelfinavir target. This approach can identify not only the drug target gene deletion strain, which could be more sensitive to the drug if the target gene is essential, but also the synthetic lethal strains to the drug if the deleted gene product interacts with the drug target either genetically or physically. Among the 17 strains with more than two-fold increase in sensitivity to nelfinavir, the majority of them are known to directly interact with HSP82, the yeast ortholog of HSP90. All of the above genetic interactions are synthetic growth defect or synthetic lethal with HSP82 (Saccharomyces Genome Database, http://www.yeastgenome.org/) (last accessed August 1, 2012), suggesting that HSP90 is a likely molecular target of nelfinavir in eukaryotic cells. Recently, another HIV protease inhibitor, ritonavir, has been reported to inhibit the functions of HSP90 (37), supporting an idea that a certain class of HIV protease inhibitors is a new HSP90 antagonist.

HSP90 is a molecular chaperone that facilitates the folding of proteins after translation or under stress conditions and has a stabilizing effect on a number of client proteins. Several specific inhibitors of HSP90 have been developed to date (38). We systematically compared the effects of nelfinavir and two known HSP90 inhibitors in a number of biochemical and cellular assays. Geldanamycin is known to bind to the N-terminal ATPbinding pocket of HSP90 and affect interaction between HSP90 and some other co-chaperones (39). Similar to geldanamycin, nelfinavir enhanced the interaction between HSP90 and HSP70. Unlike geldanamycin, which reduced the interaction of p23 with HSP90, nelfinavir had no effect on the same interaction. Moreover, geldanamycin has no effect of the HSP90 conformation changes, as reflected in trypsin digestion profiles, whereas nelfinavir substantially changed the stability and the proteolytic profile of HSP90, further distinguishing nelfinavir from geldanamycin. Novobiocin is known to bind to the C-terminal dimerization domain of HSP90, thereby inhibiting HSP90 dimerization and inducing its conformational changes $(31,33)$. Unlike novobiocin, nelfinavir had no effect on the dimerization of HSP90. Interestingly, the effects of nelfinavir on the trypsin cleavage profiles were completely abrogated by novobiocin. Although the phenotypic changes elicited by nelfinavir are largely identical to those caused by novabiocin at the cellular level, the observed difference between the two inhibitors suggest that they act differently on HSP90, likely through proximal but distinct binding sites. Although the lack of proper affinity probe of nelfinavir hampered the determination of the precise binding site of nelfinavir on HSP90, these results clearly suggest that nelfinavir interacts with a site of HSP90 distinct from the binding sites of all known inhibitors and thus represents a new type of HSP90 inhibitor.

HER2 and AKT are known client proteins of HSP90, which is required for their stability and functions $(40,41)$. Inhibition of HSP90 functions by pharmacological inhibitors leads to degradation of HER2 and AKT or a decrease in their phosphorylation $(41,42)$. In this study, nelfinavir was found to decrease the levels of total protein and phosphorylated form of HER2 and AKT in breast cancer cells, though short-term treatment (1-4 hours) showed an opposite effect. These effects of nelfinavir are attributable to its inhibition of HSP90. However, nelfinavir increased the levels of total protein and phosphorylated form of AKT in BT474 cells in animals in vivo. These results are consistent with a previous report showing that nelfinavir increased AKT phosphorylation in melanoma cells, but it inhibited the cancer cell growth (17). In a separate study, short-term treatment with nelfinavir increased AKT phosphorylation in lung cancer cells and had no inhibitory effect on AKT phosphorylation in animal tumor xenograft model, though it had a significant anticancer activity (27). These results suggest that the PI3K and AKT pathway may not be the common mechanism of anticancer activity of nelfinavir in vivo. Rather, nelfinavir exhibited anticancer activity through inhibition of multiple pathways, including Cdc25A, CDKs (17), and hypoxia inducible factor- $1 \alpha$ (43) as well as HER2 (shown in this study), all of which are known client proteins of HSP90. These results suggest that the mechanism underlying the anticancer activity of nelfinavir is the inhibition of HSP90, followed by the dysfunction of HSP90 client proteins. It is unclear, however, why AKT was increased by nelfinavir in the tumor mouse model. It is interesting to note that both nelfinavir and novobiocin increased the level of HER2 in early time points of treatment (1-4 hours) but decreased the levels after longer exposure. In contrast, geldanamycin quickly (within an hour) decreased the level of HER2 in the breast cancer cells upon treatment (data not shown). These results suggest that there is a difference between N-terminal and C-terminal HSP90 inhibitors in the kinetics of client protein stabilization and destabilization.

In addition to HER2 and AKT, a number of other client proteins of HSP90, including EGFR, HIF-1 $\alpha$, androgen receptor, Bcr-Abl, and CDKs, are known to be key players of cancer cell survival and proliferation. It has been suggested that inhibition of HSP90 function can cause simultaneous inhibitory effects on multiple pathways of cancer cell signaling, which may decrease drug resistance (44). The development of drug resistance against trastuzumab has been one of the biggest hurdles in the treatment of HER2 breast cancer. It has been shown that activating mutation in PIK3CA is the major cause of the drug resistance against HER2 targeting agents, including trastuzumab and lapatinib (45). PIK3CA encodes the $\mathrm{p} 110 \alpha$ catalytic subunit of PI3K, which is a direct downstream effecter kinase of HER2. Thus, constitutively active PI3K could allow cancer cells to bypass the effect of HER2 targeting agents. Nelfinavir, indeed, was effective in inhibiting the growth of drug-resistant, HER2-positive breast cancer cell lines in large part because a large number of signaling proteins downstream of HER2 are dependent on HSP90 to function, allowing nelfinavir to overcome resistance to both trastuzumab and lapatinib.

This work does have a few limitations. First, the precise mode of interaction between nelfinavir and HSP90 remains to be further elucidated using such techniques as x-ray crystallography. Second, we were not blinded to the control and treatment groups of animals, which may affect the objectivity of the interpretation of the experimental outcome.

Nelfinavir is a first-generation HIV protease inhibitor approved by the US Food and Drug Administration for an oral dose regimin of $750 \mathrm{mg}$ three times daily for AIDS patients. However, it was modified to a regimen of $1250 \mathrm{mg}$ twice daily, as recommended 
by US Food and Drug Administration in 1999. The efficacy of nelfinavir was proven to be equal in both regimens in a large, randomized trial (46). Extensive pharmacokinetics studies have shown that nelfinavir has an average peak plasma level of $8-10 \mu \mathrm{M}$, which is approximately twice as high as its $\mathrm{IC}_{50}$ for the HER2-positive breast cancer cell proliferation (3-6 $\mu M)$, suggesting that it may be effective in breast cancer patients with the current dosage regimen. With a relatively low toxicity profile and much available information on its drug-drug interactions and on pharmacokinetics, nelfinavir is ready for clinical testing in HER2 breast cancer patients. In conclusion, the discovery of HER2 selective inhibition of breast cancer cells by nelfinavir and the elucidation of its unique mode of action through binding to a new site on HSP90 have important implications in the development of nelfinavir and its analogs as new anticancer agents.

\section{References}

1. American Cancer Society. Breast cancer facts \& figures 2011-2012. American Cancer Society Web site. http://www.cancer.org/Research/ CancerFactsFigures/BreastCancerFactsFigures/breast-cancer-facts-andfigures-2011-2012. Accessed September 11, 2012.

2. Offit K. BRCA mutation frequency and penetrance: new data, old debate. 7 Natl Cancer Inst. 2006;98(23):1675-1677.

3. Lakhani SR, Van De Vijver MJ, Jacquemier J, et al. The pathology of familial breast cancer: predictive value of immunohistochemical markers estrogen receptor, progesterone receptor, HER-2, and $\mathrm{p} 53$ in patients with mutations in BRCA1 and BRCA2. 7 Clin Oncol. 2002;20(9):2310-2318.

4. Osborne CK. Steroid hormone receptors in breast cancer management. Breast Cancer Res Treat. 1998;51(3):227-238.

5. Stern DF. Tyrosine kinase signalling in breast cancer: ErbB family receptor tyrosine kinases. Breast Cancer Res. 2000;2(3):176-183.

6. Slamon DJ, Clark GM, Wong SG, Levin WJ, Ullrich A, McGuire WL. Human breast cancer: correlation of relapse and survival with amplification of the HER-2/neu oncogene. Science. 1987;235(4785):177-182.

7. Goldenberg MM. Trastuzumab, a recombinant DNA-derived humanized monoclonal antibody, a novel agent for the treatment of metastatic breast cancer. Clin Ther. 1999;21(2):309-318.

8. Geyer CE, Forster J, Lindquist D, et al. Lapatinib plus capecitabine for HER2-positive advanced breast cancer. $N$ Engl 7 Med. 2006;355(26):2733-2743.

9. Kim YS, Alarcon SV, Lee S, et al. Update on Hsp90 inhibitors in clinical trial. Curr Top Med Chem. 2009;9(15):1479-1492.

10. Sjoblom T, Jones S, Wood LD, et al. The consensus coding sequences of human breast and colorectal cancers. Science. 2006;314(5797):268-274.

11. Chong CR, Chen X, Shi L, Liu JO, Sullivan DJ, Jr. A clinical drug library screen identifies astemizole as an antimalarial agent. Nat Chem Biol. 2006;2(8):415-416.

12. Shim JS, Matsui Y, Bhat S, et al. Effect of nitroxoline on angiogenesis and growth of human bladder cancer. 7 Natl Cancer Inst. 2010;102(24):1855-1873.

13. James JS. Nelfinavir (Viracept) approved: fourth protease inhibitor available. AIDS Treat News. 1997; 1997(267):1-2.

14. Bardsley-Elliot A, Plosker GL. Nelfinavir: an update on its use in HIV infection. Drugs. 2000;59(3):581-620.

15. Garofalo RS, Orena SJ, Rafidi K, et al. Severe diabetes, age-dependent loss of adipose tissue, and mild growth deficiency in mice lacking Akt2/PKB beta. F Clin Invest. 2003;112(2):197-208.

16. Yang Y, Ikezoe T, Nishioka C, et al. NFV, an HIV-1 protease inhibitor, induces growth arrest, reduced Akt signalling, apoptosis and docetaxel sensitisation in NSCLC cell lines. Br 7 Cancer: 2006;95(12):1653-1662.

17. Jiang W, Mikochik PJ, Ra JH, et al. HIV protease inhibitor nelfinavir inhibits growth of human melanoma cells by induction of cell cycle arrest. Cancer Res. 2007;67(3):1221-1227.

18. Schuurbiers OC, Kaanders JH, van der Heijden HF, Dekhuijzen RP, Oyen WJ, Bussink J. The PI3-K/AKT-pathway and radiation resistance mechanisms in non-small cell lung cancer. 7 Thorac Oncol. 2009;4(6):761-767.

19. Zhang T, Cui GB, Zhang J, et al. Inhibition of PI3 kinases enhances the sensitivity of non-small cell lung cancer cells to ionizing radiation. Oncol Rep. 2010;24(6):1683-1689.

20. Gupta AK, Cerniglia GJ, Mick R, et al. Radiation sensitization of human cancer cells in vivo by inhibiting the activity of PI3K using LY294002. Int 7 Radiat Oncol Biol Phys. 2003;56(3):846-853.

21. Brunner TB, Geiger M, Grabenbauer GG, et al. Phase I trial of the human immunodeficiency virus protease inhibitor nelfinavir and chemoradiation for locally advanced pancreatic cancer. 7 Clin Oncol. 2008;26(16): 2699-2706.

22. Piccinini M, Rinaudo MT, Anselmino A, et al. The HIV protease inhibitors nelfinavir and saquinavir, but not a variety of HIV reverse transcriptase inhibitors, adversely affect human proteasome function. Antivir Ther: 2005;10(2):215-223.

23. Gupta AK, Li B, Cerniglia GJ, Ahmed MS, Hahn SM, Maity A. The HIV protease inhibitor nelfinavir downregulates Akt phosphorylation by inhibiting proteasomal activity and inducing the unfolded protein response. Neoplasia. 2007;9(4):271-278.

24. Bernstein WB, Dennis PA. Repositioning HIV protease inhibitors as cancer therapeutics. Curr Opin HIV AIDS. 2008;3(6):666-675.

25. Rowe DL, Ozbay T, Bender LM, Nahta R. Nordihydroguaiaretic acid, a cytotoxic insulin-like growth factor-I receptor/HER2 inhibitor in trastuzumab-resistant breast cancer. Mol Cancer Ther. 2008;7(7):1900-1908.

26. Chong CR, Xu J, Lu J, Bhat S, Sullivan DJ, Jr, Liu JO. Inhibition of angiogenesis by the antifungal drug itraconazole. ACS Chem Biol. 2007;2(4):263-270.

27. Gills JJ, Lopiccolo J, Tsurutani J, et al. Nelfinavir, a lead HIV protease inhibitor, is a broad-spectrum, anticancer agent that induces endoplasmic reticulum stress, autophagy, and apoptosis in vitro and in vivo. Clin Cancer Res. 2007;13(17):5183-5194.

28. Wakeling AE, Guy SP, Woodburn JR, et al. ZD1839 (Iressa): an orally active inhibitor of epidermal growth factor signaling with potential for cancer therapy. Cancer Res. 2002;62(20):5749-5754.

29. Kim D, Cheng GZ, Lindsley CW, Yang H, Cheng JQ. Targeting the phosphatidylinositol-3 kinase/Akt pathway for the treatment of cancer. Curr Opin Investig Drugs. 2005;6(12):1250-1258.

30. Hartson SD, Thulasiraman V, Huang W, Whitesell L, Matts RL. Molybdate inhibits hsp90, induces structural changes in its C-terminal domain, and alters its interactions with substrates. Biocbemistry. 1999;38(12): 3837-3849.

31. Yun BG, Huang W, Leach N, Hartson SD, Matts RL. Novobiocin induces a distinct conformation of Hsp90 and alters Hsp90-cochaperone-client interactions. Biochemistry. 2004;43(25):8217-8229.

32. Yin Z, Henry EC, Gasiewicz TA. (-)-Epigallocatechin-3-gallate is a novel Hsp90 inhibitor. Biochemistry. 2009;48(2):336-345.

33. Allan RK, Mok D, Ward BK, Ratajczak T. Modulation of chaperone function and cochaperone interaction by novobiocin in the C-terminal domain of Hsp90: evidence that coumarin antibiotics disrupt Hsp90 dimerization. f Biol Chem. 2006;281(11):7161-7171.

34. Chong CR, Qian DZ, Pan F, et al. Identification of type 1 inosine monophosphate dehydrogenase as an antiangiogenic drug target. $7 \mathrm{Med}$ Chem. 2006;49(9):2677-2680.

35. Ren YR, Pan F, Parvez S, et al. Clofazimine inhibits human Kv1.3 potassium channel by perturbing calcium oscillation in T lymphocytes. PLoS One. 2008;3(12):e4009.

36. Xu J, Dang Y, Ren YR, Liu JO. Cholesterol trafficking is required for mTOR activation in endothelial cells. Proc Natl Acad Sci U S A. 2010;107(10):4764-4769.

37. Srirangam A, Mitra R, Wang M, et al. Effects of HIV protease inhibitor ritonavir on Akt-regulated cell proliferation in breast cancer. Clin Cancer Res. 2006;12(6):1883-1896.

38. Trepel J, Mollapour M, Giaccone G, Neckers L. Targeting the dynamic HSP90 complex in cancer. Nat Rev Cancer. 2010;10(8):537-549.

39. Zhang T, Hamza A, Cao X, et al. A novel Hsp90 inhibitor to disrupt Hsp90/ Cdc37 complex against pancreatic cancer cells. Mol Cancer Ther. 2008;7(1): $162-170$. 
40. Sato S, Fujita N, Tsuruo T. Modulation of Akt kinase activity by binding to Hsp90. Proc Natl Acad Sci U S A. 2000;97(20):10832-10837.

41. Citri A, Alroy I, Lavi S, et al. Drug-induced ubiquitylation and degradation of ErbB receptor tyrosine kinases: implications for cancer therapy. $E M B O$ 7. 2002;21(10):2407-2417.

42. Basso AD, Solit DB, Chiosis G, Giri B, Tsichlis P, Rosen N. Akt forms an intracellular complex with heat shock protein 90 (Hsp90) and Cdc37 and is destabilized by inhibitors of Hsp90 function. 7 Biol Chem. 2002;277(42):39858-39866.

43. Pore N, Gupta AK, Cerniglia GJ, et al. Nelfinavir down-regulates hypoxiainducible factor 1alpha and VEGF expression and increases tumor oxygenation: implications for radiotherapy. Cancer Res. 2006;66(18): 9252-9259.

44. Workman P, Burrows F, Neckers L, Rosen N. Drugging the cancer chaperone HSP90: combinatorial therapeutic exploitation of oncogene addiction and tumor stress. Ann NY Acad Sci. 2007;1113:202-216.

45. Berns K, Horlings HM, Hennessy BT, et al. A functional genetic approach identifies the PI3K pathway as a major determinant of trastuzumab resistance in breast cancer. Cancer Cell. 2007;12(4):395-402.

46. Marzolini C, Buclin T, Decosterd LA, Biollaz J, Telenti A. Nelfinavir plasma levels under twice-daily and three-times-daily regimens: high interpatient and low intrapatient variability. Ther Drug Monit. 2001;23(4): 394-398.

\section{Funding}

National Cancer Institute (CA122814); Flight Attendant Medical Research Institute (FAMRI); the Commonwealth Foundation (to JOL); the National Institutes of Health (NIH) (R01AI065983 to RR); National Center for Research Resources, a component of the NIH and NIH Roadmap for Medical Research (UL1 RR 025005 to Johns Hopkins School of Medicine).

\section{Notes}

We are grateful to Dr Bert Vogelstein for kindly providing the breast cancer cell lines. We thank Dr Solomon Snyder for providing HSP90 $\alpha$ construct. The authors are solely responsible for the design of the study, the analysis and interpretation of the data, the writing of the manuscript, and the decision to submit the manuscript for publication.

Affiliations of authors: Department of Pharmacology and Molecular Sciences (JSS, JOL), Department of Oncology (JOL), and Department of Physiology (RR), Johns Hopkins School of Medicine, and Department of Environmental Health Sciences $(\mathrm{IH})$, Johns Hopkins Bloomberg School of Public Health, Baltimore, MD; Urologic Oncology Branch, Center for Cancer Research, National Cancer Institute, Bethesda, MD (KB, LN); Department of Pharmacology, Emory University School of Medicine, Atlanta, GA (RN). 